\title{
Holographic spontaneous parity breaking and emergent hall viscosity and angular momentum
}

\author{
Dam Thanh Son and Chaolun Wu \\ Kadanoff Center for Theoretical Physics and Enrico Fermi Institute, University of Chicago, \\ Chicago, Illinois 60637, U.S.A. \\ E-mail: dtson@uchicago.edu, chaolunwu@uchicago.edu
}

ABSTRACT: We study the spontaneous parity breaking and generating of Hall viscosity and angular momentum in holographic p+ip model, which can describe strongly-coupled chiral superfluid states in many quantum systems. The dual gravity theory, an SU(2) gauge field minimally coupled to Einstein gravity, is parity-invariant but allows a black hole solution with vector hair corresponding to a parity-broken superfluid state. We show that this state possesses a non-vanishing parity-odd transport coefficient - Hall viscosity - and an angular momentum density. We first develop an analytic method to solve this model near the critical regime and to take back-reactions into account. Then we solve the equation for the tensor mode fluctuations and obtain the expression for Hall viscosity via Kubo formula. We also show that a non-vanishing angular momentum density can be obtained through the vector mode fluctuations and the corresponding boundary action. We give analytic results of both Hall viscosity and angular momentum density near the critical regime in terms of physical parameters. The near-critical behavior of Hall viscosity is different from that obtained from a gravitational Chern-Simons model. We find that the magnitude of Hall viscosity to angular momentum density ratio is numerically consistent with being equal to $1 / 2$ at large $\mathrm{SU}(2)$ coupling corresponding to the probe limit, in agreement with previous results obtained for various quantum fluid systems and from effective theory approaches. In addition, we find the shear viscosity to entropy density ratio remains above the universal bound.

Keywords: AdS-CFT Correspondence, Holography and condensed matter physics (AdS/CMT), Spontaneous Symmetry Breaking, Discrete and Finite Symmetries

ArXiv EPRINT: 1311.4882 


\section{Contents}

1 Introduction 1

2 Relativistic first order parity-violating hydrodynamics 4

3 Einstein-SU(2) system $\quad 6$

$\begin{array}{lll}3.1 \text { Bulk and boundary actions } & 6\end{array}$

$\begin{array}{lll}3.2 & \text { Perturbative expansion of actions and EOMs } & 7\end{array}$

4 Holographic $p_{x}+i p_{y}$ model $\quad 8$

4.1 Background and its symmetries 8

$\begin{array}{ll}4.2 \text { Boundary conditions and thermodynamical functions } & 10\end{array}$

$\begin{array}{lll}4.3 & \text { Background on-shell action } & 11\end{array}$

$\begin{array}{lll}4.4 & \text { AdS-Reissner-Nordström solution } & 12\end{array}$

$\begin{array}{ll}\text { 4.5 Analytic approach to the symmetry-breaking solution } & 12\end{array}$

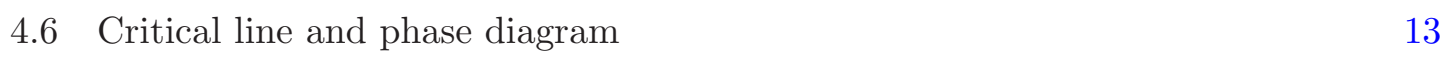

$\begin{array}{lll}4.7 & \text { Near-critical behavior of order parameter } & 16\end{array}$

4.8 Near-critical behaviors of charge density and chemical potential 19

4.9 Near-critical behaviors of characteristic functions 20

5 Tensor mode fluctuations and viscosities $\quad 21$

$\begin{array}{lll}5.1 & \text { Mode classification } & 21\end{array}$

$\begin{array}{ll}5.2 & \text { Tensor mode EOMs } \\ 5.3 & \text { Bous }\end{array}$

5.3 Boundary-to-bulk propagators 23

$\begin{array}{ll}5.4 \text { 2-point functions and viscosities } & 25\end{array}$

$\begin{array}{ll}5.5 & \text { Bulk-to-bulk propagators }\end{array}$

$\begin{array}{ll}5.6 & \text { Hall viscosity } \\ & 29\end{array}$

6 Vector mode fluctuations and angular momentum $\quad 30$

6.1 Edge current and angular momentum density 30

$\begin{array}{lll}6.2 & \text { Linear on-shell action } & 31\end{array}$

6.3 Vector mode EOMs 32

6.4 Boundary-to-bulk propagators 33

6.5 Angular momentum density and ratio to Hall viscosity 34

7 Low temperature limit $\quad \mathbf{3 5}$

$\begin{array}{ll}7.1 \text { About the complete phase diagram } & 35\end{array}$

7.2 Near-extremal AdS-RN black hole with condensate 37

$\begin{array}{lll}7.3 & \text { Extremal AdS-RN black hole with condensate } & 37\end{array}$

8 Conclusions and comments $\quad 39$ 


\section{Introduction}

Systems with broken parity and time-reversal symmetries have long been attractive and active fields to both experimentalists and theorists in physics. When these discrete symmetries are allowed to be broken, additional transport coefficients can arise in the hydrodynamic description of the systems. In $2+1$-dimensional systems, Hall conductivity, the parity-odd and dissipationless counterpart of the ordinary longitudinal conductivity, is probably the most famous and best-studied example. It is also been known that the viscosity can have a parity-odd and dissipationless part as well, called Hall viscosity. On contrary to the parity-even and dissipative shear viscosity, which, in classical picture of fluids, tends to accelerate or decelerate nearby flows in the presence of a gradient of velocity, Hall viscosity tends to repel or attract the nearby flows. The underlying force (sometimes referred to as "Lorentz shear force" in the literature) is perpendicular to the flow, thus is dissipationless. A pictorial illustration can be found in [1]. An example of Hall viscosity in classical fluid systems is given by a plasma moving in magnetic field ([2], eq. (59.38)). Recently, Hall viscosity was studied for various non-relativistic quantum systems such as quantum Hall fluids [3-9] and chiral superfluids and superconductors [7, 9], and relativistic quantum systems such as topological insulators with massive Dirac fermions $[1,10]$. It was also studied using general approaches such as linear response theory [11], effective field theories [12-15], viscoelastic-electromagnetism [16] and quantum hydrodynamics of vortex flow [17-19]. Hall viscosity possesses many interesting properties. In quantum Hall fluids it is related to the Berry curvature and the Wen-Zee shift [20], thus reflects the topological feature of the quantum Hall states. Hall viscosity also enters as a finite wave number correction to Hall conductivity [12]. Of particular interest is a general relation between Hall viscosity $\eta_{H}$ and angular momentum density $\ell$ of the system:

$$
\eta_{H}=-\frac{1}{2} \ell
$$

which is derived first in [7] for quantum Hall states and $p_{x}+i p_{y}$ superfluids, then in [13-15] from effective field theory methods. In this paper we try to understand Hall viscosity and the above relation to angular momentum density in strongly-interacting quantum manybody systems, particularly the $p_{x}+i p_{y}$ paired states, from the holographic point of view.

Over the last decade, holography, or gauge/gravity duality [21-23] has been widely applied to study many strongly interacting systems. One of its remarkable early successes is to study hydrodynamic transport coefficients of strongly coupled relativistic conformal fluids $[24,25]$, in particular the shear viscosity to entropy density ratio [26] (for an recent 
review, see [27]), whose theoretical value obtained via holography is very close to that of the quark-gluon plasma extracted from RHIC and LHC data.

Another recent application of holography is on superconducting and superfluid phase transitions in condensed matter systems. The hope is to gain insight into systems that cannot be described by the BCS theory, like high $T_{c}$ superconductors. The superconducting phase transition is characterized by a charged operator $\mathcal{O}$, whose expectation value $\langle\mathcal{O}\rangle$ is zero above a certain critical temperature $T_{c}$ (the normal phase), but becomes non-zero below $T_{c}$ (the superconducting phase). In the dual gravity theory, the normal phase at non-zero temperature is usually described by a charged AdS black hole. The operator $\mathcal{O}$ is dual to a certain charged matter field $\phi$ that couples to this black hole. Below the critical temperature $T_{c}, \phi$ can develop a non-trivial profile outside the black hole horizon [28, 29]. The resulting hairy black hole, which is thermodynamically preferred over the hairless one, describes the superconducting phase. Depending on whether the matter field $\phi$ is a scalar, a non-Abelian gauge field, or a symmetric tensor field, it describes holographically the $s$-wave [30-33], $p$-wave [34-36] or $d$-wave [37, 38] superconductors respectively. In [31] it is shown that such holographic superconductors are Type II superconductors. For reviews in this subject, see [39, 40].

A third application of holography is to strongly coupled systems with broken parity and time-reversal symmetries, such as quantum Hall systems [41-45]. For 2+1-dimensional systems, dyonic AdS black hole is a simple holographic realization of the classical Hall effect and produces unquantized Hall conductivity [46]. To address quantum Hall effects, including the integer and fractional quantized Hall conductivities, quantum plateau transitions and edge states, models with various matter fields or brane configurations were considered [47-52], and these added structures usually include Chern-Simons terms, which break the parity of the theories explicitly or spontaneously. In $3+1$ dimensions, two other parity-violating effects - the chiral magnetic effect and chiral vortical effect - are also realized in holographic models [53-61].

Hall viscosity was obtained in [62] for the first time in a holographic model, with a dynamical axion coupled to Chern-Simons modified gravity [63], and numerics was soon followed $[64,65]$. Refs. [66, 67] studied both Hall viscosity and Curl viscosity using similar holographic models with Chern-Simons terms. Angular momentum generated in holographic models was also studied [68, 69]. However, whether a holographic model can generate both Hall viscosity and angular momentum density simultaneously and whether their relation (1.1) can hold remain mysterious. For example, for the models considered in both [70] and [68] there exists an angular momentum but no Hall viscosity.

What we study in this paper is an overlap of all the aforementioned areas in holography. We will show that for the holographic $p_{x}+i p_{y}$ model of [34], in the superconducting phase, both non-vanishing Hall viscosity and angular momentum density emerge. Using analytic method to compute both of them near the critical regime, we find that the relation (1.1) holds at the probe limit regime, but has a deviation when back-reactions are taken into account. This model is different from most other holographic models constructed for Hall effects and those used in $[62,68]$ to compute Hall viscosity and angular momentum: it does not contain an explicit Chern-Simons term in the action, nor external magnetic field 
or rotation [71], which all break parity and time-reversal symmetry in a manifest way. It is known that in some types of superconducting phase transitions the breaking of $U(1)$ symmetry is accompanied by a spontaneous parity breaking. In the field theory picture of this model, the parity is broken spontaneously below the critical temperature by the formation of $p_{x}+i p_{y}$ paired ground state of the BCS theory. The total orbital angular momentum of the Cooper pairs is at eigenstate $|l m\rangle=|11\rangle$, which breaks parity and time-reversal symmetry. In the dual gravity theory which involves Einstein gravity and $\mathrm{SU}(2)$ gauge field, the parity is broken by the $\mathrm{SU}(2)$ hair of the black hole, which is dual to the $p_{x}+i p_{y}$ paired ground state. This particular background locks the $\mathbb{Z}_{2}$ symmetry of spatial reflection in the two conformally flat spatial directions to the $\mathbb{Z}_{2}$ symmetry in the $\mathrm{SU}(2)$ vector space. The $\mathrm{SU}(2)$ gauge connection term breaks the latter $\mathbb{Z}_{2}$ symmetry explicitly, and this breaking is propagated to the former spatial $\mathbb{Z}_{2}$ symmetry through the background. This finally produces non-trivial parity-breaking effects such as the emergence of Hall conductivity and angular momentum density, and as expected, they are both proportional to the $\mathrm{SU}(2)$ gauge coupling. The $p_{x}+i p_{y}$ model has a gapped zero temperature ground state, which also ensures that the dissipationless transport can take place and Hall viscosity is non-vanishing. The gap energy and thermal Hall conductivity was numerically calculated in [36].

The holographic $p_{x}+i p_{y}$ model of [34] was previously studied mostly in the context of superconductivity and superfluidity. However it is worth to note here that it may have richer physics yet to explore, for example, the parity-breaking effects on transport studied in [36] and this paper. In fact the $p_{x}+i p_{y}$ model is more than just a description of chiral superfluid states in, for example, the A-phase of Helium-3 [72-74] and layered $\mathrm{Sr}_{2} \mathrm{RuO}_{4}$ superconductors $[75,76]$. It also plays an important role in understanding the $\nu=5 / 2$ quantum Hall state and all interesting physics associated with it, such as non-Abelian anyons and its potential application to quantum computation (for recent reviews on this subject, see [77-79]).

It is worth noting here that [35] shows the $p_{x}+i p_{y}$ superconducting state can be unstable upon perturbations and tunnel to an anisotropic $p$-wave ground state. However, this problem might be overcome by adding non-linear terms in the action to stabilize the $p_{x}+i p_{y}$ solution, similar as in [80]. As long as the solution remains homogeneous and isotropic, the parity breaking properties studied in this paper will still hold qualitatively in the new model, with added corrections from the non-linear effect.

The paper is organized as following. In section 2, we briefly review first order relativistic parity-violating hydrodynamics, including the definition of Hall viscosity in this context and Kubo formulae associated to it. In section 3 we give the general formalism of Einstein$\mathrm{SU}(2)$ system, which is the basis where the holographic $p$-wave superconductor models are built on. In section 4 we review the $p_{x}+i p_{y}$ model of [34] and propose our refined analytic method to solve this model near the critical regime. Our method takes the backreactions between the metric and the matter field into full consideration. In the next two sections we compute Hall viscosity and angular momentum density from tensor and vector mode bulk fluctuations respectively, and then the ratio between them. In section 7 the low temperature limit of the model is investigated. In the last section we will make conclusion 
remarks and comments. Except that in section 3 that we work in general $d+1$ dimensions, we always work in $3+1$ dimensions in the gravity theory, thus the dual field theory or condensed matter systems are in $2+1$ dimensions.

\section{Relativistic first order parity-violating hydrodynamics}

Hydrodynamics is a large-scale effective description of fluids and many other classical and quantum systems at non-zero temperature. The fundamental EOMs are the conservation of the energy-stress tensor and the current:

$$
\begin{aligned}
\nabla_{\mu} T^{\mu \nu} & =F^{\nu \mu} J_{\mu} \\
\nabla_{\mu} J^{\mu} & =0
\end{aligned}
$$

Here we allow the current to couple to an external gauge field whose strength is $F_{\mu \nu}$. For simplicity we assume there is only a U(1) symmetry associated with the conserved current. This is obviously not true like in the case of $\mathrm{SU}(2)$ gauge symmetry considered in the rest of this paper. In that case it is straightforward to generalize by letting the current and transport coefficients associated with it carry $\mathrm{SU}(2)$ vector indices, but the Kubo formulae for viscosities will remain the same. When the system has conformal symmetry, there is an additional equation of state due to scale invariance:

$$
T_{\mu}^{\mu}=0
$$

This is the case we will study in this paper. To solve the above equations for a particular system, one need to supplement them with constitutive relations which specify the form of $T^{\mu \nu}$ and $J^{\mu}$ in terms of derivative expansion of local macroscopic functions such as energy density, pressure and velocity field, among others. Terms allowed in these constitutive relations can be determined based on symmetries of the systems and thermodynamical considerations, up to some arbitrary constants to be determined by the underlying microscopic theory. These constants are the transport coefficients. For relativistic conformal systems, there is only one possible first order term allowed by symmetries in each of $T^{\mu \nu}$ and $J^{\mu}$, whose coefficients are the shear viscosity and conductivity, respectively.

When parity is not respected, there are additional terms allowed in the constitutive relations, with additional transport coefficients. Based on symmetries and thermodynamic considerations, [70] systematically studied this case for relativistic fluid in $2+1$ dimensions and obtained complete first order constitutive relations (the non-relativistic version was also studied recently in [81]). In this paper, we are interested in the sourceless case when external $F^{\mu \nu}=0$, and for simplicity we also assume that the temperature $T$ and chemical potential $\mu$ are not local functions. The constitutive relations up to first order in derivatives are

$$
\begin{aligned}
T^{\mu \nu} & =\varepsilon u^{\mu} u^{\nu}+\left(p-\zeta \nabla_{\alpha} u^{\alpha}-\zeta_{H} \Omega\right) \Delta^{\mu \nu}-\eta \sigma^{\mu \nu}-\eta_{H} \tilde{\sigma}^{\mu \nu}, \\
J^{\mu} & =\rho u^{\mu} .
\end{aligned}
$$


The velocity field is normalized to $u^{\mu} u_{\mu}=-1$ and

$$
\begin{aligned}
\Delta^{\mu \nu} & =g^{\mu \nu}+u^{\mu} u^{\nu} \\
\sigma^{\mu \nu} & =\Delta^{\mu \alpha} \Delta^{\nu \beta}\left(\nabla_{\alpha} u_{\beta}+\nabla_{\beta} u_{\alpha}-g_{\alpha \beta} \nabla_{\gamma} u^{\gamma}\right), \\
\tilde{\sigma}^{\mu \nu} & =\frac{1}{2}\left(\epsilon^{\mu \alpha \beta} u_{\alpha} \sigma_{\beta}{ }^{\nu}+\epsilon^{\nu \alpha \beta} u_{\alpha} \sigma_{\beta}{ }^{\mu}\right) \\
\Omega & =-\epsilon^{\mu \nu \alpha} u_{\mu} \nabla_{\nu} u_{\alpha} .
\end{aligned}
$$

In the above definitions, the expression for shear flow $\sigma^{\mu \nu}$ quoted here is only valid in $2+1$ dimensions. For general $d$-dimensional spacetime, the last term will have a coefficient $-\frac{2}{d-1}$ rather than -1 . The definitions of $\tilde{\sigma}^{\mu \nu}$ and $\Omega$ are only possible for $2+1$ dimensions because the rank-3 totally anti-symmetric tensor $\epsilon^{\mu \nu \alpha}$ only exists in this case. An analogous case in $d=4$ has also been studied, for example, in [57,61]. The coefficients $\zeta, \eta$ and $\eta_{H}$ are bulk, shear and Hall viscosities and $\varepsilon, p$ and $\rho$ are energy density, pressure and charge density of the system. For a conformal system, $\nabla_{\alpha} u^{\alpha}$ and $\Omega$ parts will drop off, so $\zeta=\zeta_{H}=0$, and this is the case we will consider in this paper. A double perturbative expansion in derivatives and metric fluctuations [62] gives

$$
T^{x y}=-p h_{x y}-\eta \frac{\partial}{\partial t} h_{x y}+\frac{1}{2} \eta_{H} \frac{\partial}{\partial t}\left(h_{x x}-h_{y y}\right)+O\left(\vec{\partial}, h^{2}\right),
$$

where $h_{\mu \nu}$ is the metric fluctuation around the flat Minkowskian background metric and the coordinates are $x^{\mu}=(t, x, y)$. Using

$$
\left\langle T^{\mu \nu}(x)\right\rangle_{h}=\left\langle T^{\mu \nu}(x)\right\rangle_{h=0}-\frac{1}{2} \int d^{3} x^{\prime} G_{\mathrm{ra}}^{\mu \nu, \alpha \beta}\left(x, x^{\prime}\right) h_{\alpha \beta}\left(x^{\prime}\right)+O\left(h^{2}\right),
$$

where the causal 2-point functions of energy-stress tensor in position space are defined as

$$
G_{\mathrm{ra}}^{\mu \nu, \alpha \beta}\left(x, x^{\prime}\right)=-i \theta\left(t-t^{\prime}\right)\left\langle\left[T^{\mu \nu}(x), T^{\alpha \beta}\left(x^{\prime}\right)\right]\right\rangle
$$

and those in momentum space are defined as

$$
G_{\mathrm{ra}}^{\mu \nu, \alpha \beta}(k)=\int d^{3} x e^{-i k x} G_{r a}^{\mu \nu, \alpha \beta}(x, 0)
$$

with momentum $k^{\mu}=(\omega, \vec{k})$, we obtain the hydrodynamic expansions for the following 2-point functions

$$
\begin{aligned}
G_{\mathrm{ra}}^{x y, x x-y y}(\omega, \vec{k}=0) & =2 i \eta_{H} \omega+O\left(\omega^{2}\right), \\
G_{\mathrm{ra}}^{x y, x y}(\omega, \vec{k}=0) & =p-i \eta \omega+O\left(\omega^{2}\right) .
\end{aligned}
$$

These will then give the Kubo formulae for the viscosities.

At this point it is reasonable to ask whether the above formulae are valid and can be applied to the calculation of holographic $p_{x}+i p_{y}$ model. There are two subtleties. The first one is regarding the global symmetry. Clearly, the above analysis and that of [70] assume only a global U(1) gauge symmetry, but the model to be discussed in the rest of this paper has an $\mathrm{SU}(2)$ global symmetry. Some terms in the above equations, 
particularly those involving electromagnetic response in the conservation equations and hydrodynamic expansion, will change once the global gauge group is changed. For example, both the current $J^{\mu}$ and field strength $F_{\mu \nu}$ will be SU(2)-valued now. But the part involving gravitational response, such as $u^{\mu}, \sigma^{\mu \nu}$ and $\tilde{\sigma}^{\mu \nu}$, will not change. The only assumption lying behind (2.10), (2.14) and (2.15) are homogeneity and isotropy, not the global gauge symmetry. A further subtlety is that the ground state of holographic $p_{x}+i p_{y}$ model, (4.1), seems to break the apparent rotational invariance and spoil isotropy. As explained in the text below (4.1), this apparent "breaking" of spatial rotational symmetry is restored by combining the rotational symmetry of the SU(2) gauge group. Since the energy-stress tensor is an $\mathrm{SU}(2)$ singlet which does not see the rotation in the gauge group, the isotropy is preserved in the hydrodynamic analysis involving only the energy-stress tensor. Thus the formulae (2.14) and (2.15) are still valid.

The second subtlety is about the extra degrees of freedom in superfluids, namely the superfluid velocity $\xi_{\mu}=\partial_{\mu} \varphi-A_{\mu}$, where $\varphi$ is the Goldstone boson. On contrary, the velocity $u^{\mu}$ we introduce before is the normal fluid velocity. In general, they are both non-vanishing and point on different directions in the lab frame, thus there are additional first order derivative terms involving $\xi^{\mu}$ that can be added to constitutive relations of $T^{\mu \nu}$ and $J^{\mu}$ and give rise to new transport coefficients and possibly modify the existing Kubo formulae as well. Superfluid hydrodynamics in $3+1$ dimensions has been studied in [82-84] and that in $\mathrm{d}+1$ dimensions with Lifshitz scaling recently in [85] and in [86] for 2+1-dimensional non-Abelian case. Ref. [86] shows that for the case relevant to ours, the SU(2) superfluids, the Kubo formula for Hall viscosity (2.14) remains valid. However, [86] also shows that there are additional first order transport coefficients due to superfluid velocity, i.e. what they call $\tilde{\eta}_{H}$, the "locking dependent Hall viscosity", and $\kappa_{H}$, whose Kubo formulae are given by 2-point functions of energy-stress tensor and SU(2) current. The $\tilde{\eta}_{H}$ is different from the Hall viscosity $\eta_{H}$ that is studied in the rest of this paper and in the previous literature, thus will not be further considered in this paper, even though this quantity itself is interesting on its own and deserves further study. We will concentrate on the Hall viscosity given by (2.14).

The holographic prescription for computing causal 2-point functions had been studied in [87] and that for higher n-point functions in [88, 89]. In the rest of this paper we will follow those prescriptions to compute the above two 2-point functions for holographic $p_{x}+i p_{y}$ superconductor model of [34] and obtain the viscosities in that model.

\section{Einstein-SU(2) system}

\subsection{Bulk and boundary actions}

In this section, for generality we will work in $(d+1)$-dimensional curved spacetime. $z$ is the radial coordinate and $z=\infty$ is where the $d$-dimensional time-like boundary locates. The bulk action for Einstein-SU(2) system is

$$
S_{\text {bulk }}=\frac{1}{2 \kappa^{2}} \int d^{d+1} x \sqrt{-g}\left\{\mathcal{R}-2 \Lambda-\frac{1}{4}\left(F_{\mu \nu}^{\mathbf{I}}\right)^{2}\right\}
$$


where the cosmological constant $\Lambda=-\frac{d(d-1)}{2 R^{2}}$ and $R$ the AdS radius. The $\mathrm{SU}(2)$ field strength is

$$
F_{\mu \nu}^{\mathbf{I}}=\partial_{\mu} A_{\nu}^{\mathbf{I}}-\partial_{\nu} A_{\mu}^{\mathbf{I}}+\lambda \epsilon^{\mathbf{I J K}} A_{\mu}^{\mathbf{J}} A_{\nu}^{\mathbf{K}},
$$

where $\lambda$ is the Yang-Mills coupling, $\mathbf{I}, \mathbf{J}, \mathbf{K}=\mathbf{1}, \mathbf{2}, \mathbf{3}$ and $\epsilon^{\mathbf{I J K}}$ is the totally antisymmetric tensor with $\epsilon^{\mathbf{1 2 3}}=1$. The boundary terms include the Gibbons-Hawking term

$$
S_{\mathrm{GH}}=\frac{1}{\kappa^{2}} \int_{z=\infty} d^{d} x \sqrt{-\gamma} K
$$

and a counter term

$$
S_{\mathrm{ct}}=-\frac{d-1}{\kappa^{2} R} \int_{z=\infty} d^{d} x \sqrt{-\gamma},
$$

where $\hat{n}_{\mu}$ is the outgoing unit normal 1-form of the boundary, $\gamma_{\mu \nu}=g_{\mu \nu}-\hat{n}_{\mu} \hat{n}_{\nu}$ is the induced metric on the boundary and $K=\nabla_{\mu} \hat{n}^{\mu}$ is the extrinsic curvature of the boundary. To compute first order hydrodynamics this single counter term is enough. For higher order hydrodynamics, one need to include more counter terms such as the boundary Ricci scalar etc. [90-92].

\subsection{Perturbative expansion of actions and EOMs}

To compute 2-point functions, we perturbatively expand the on-shell actions around the background up to second order in field fluctuations. The metric and gauge fields are

$$
\begin{aligned}
g_{\mu \nu} & =\bar{g}_{\mu \nu}+h_{\mu \nu}, \\
A_{\mu}^{\mathbf{I}} & =\bar{A}_{\mu}^{\mathbf{I}}+a_{\mu}^{\mathbf{I}},
\end{aligned}
$$

where $\bar{g}_{\mu \nu}$ and $\bar{A}_{\mu}^{\mathbf{I}}$ are the background and $h_{\mu \nu}$ and $a_{\mu}^{\mathbf{I}}$ are fluctuations. To fully consider the back-reactions of the gauge fields on the metric, we assume $h_{\mu \nu}$ and $a_{\mu}^{\mathbf{I}}$ are of the same order. The first order on-shell action which is linear in fluctuations is

$$
S_{\text {bulk }}^{(1)}=\frac{1}{2 \kappa^{2}} \int d^{d+1} x \partial_{\mu}\left\{\sqrt{-\bar{g}}\left(\bar{\nabla}_{\nu} h^{\mu \nu}-\bar{\nabla}^{\mu} h-\bar{F}^{\mathbf{I} \mu \nu} a_{\nu}^{\mathbf{I}}\right)\right\}
$$

The second order on-shell action quadratic in fluctuations is ${ }^{1}$

$$
\begin{aligned}
S_{\text {bulk }}^{(2)}= & \frac{1}{4 \kappa^{2}} \int d^{d+1} x \partial_{\mu}\left\{\sqrt { - \overline { g } } \left[\frac{1}{2} h \bar{\nabla}_{\nu} h^{\mu \nu}+\frac{3}{2} h^{\mu \nu} \bar{\nabla}_{\nu} h-h^{\rho \sigma} \bar{\nabla}_{\rho} h_{\sigma}^{\mu}-2 h^{\mu \rho} \bar{\nabla}^{\sigma} h_{\rho \sigma}\right.\right. \\
& \left.+\frac{3}{2} h^{\rho \sigma} \bar{\nabla}^{\mu} h_{\rho \sigma}-\frac{1}{2} h \bar{\nabla}^{\mu} h-a_{\nu}^{\mathbf{I}}\left(\frac{1}{2} \bar{F}^{\mathbf{I} \mu \nu} h+\bar{F}_{\rho}^{\mathbf{I}}\left[\mu h^{\nu] \rho}+F^{\mathbf{I}(1) \mu \nu}\right)\right]\right\} .
\end{aligned}
$$

Here all co-variant derivative $\bar{\nabla}$ and raising and lowering indices are with respect to the background metric $\bar{g}_{\mu \nu}$, with $h \equiv h_{\mu}^{\mu}$ and

$$
F_{\mu \nu}^{\mathbf{I}(1)}=\bar{\nabla}_{[\mu} a_{\nu]}^{\mathbf{I}}+\lambda \epsilon^{\mathbf{I J K}} \bar{A}_{[\mu}^{\mathbf{J}} a_{\nu]}^{\mathbf{K}} .
$$

\footnotetext{
${ }^{1}$ In this paper we define the symmetrization $A_{(\mu} B_{\nu)} \equiv A_{\mu} B_{\nu}+A_{\nu} B_{\mu}$ and the anti-symmetrization $A_{[\mu} B_{\nu]} \equiv A_{\mu} B_{\nu}-A_{\nu} B_{\mu}$ without the factor of $\frac{1}{2}$.
} 
These actions are written as integrals of total derivatives, which means they are boundary terms. Choosing the gauge condition $\bar{g}_{\mu z}=0$ for $\mu \neq z$ and $h_{\mu z}=0$ for any $\mu$, the first order boundary actions are

$$
\begin{aligned}
S_{\mathrm{GH}}^{(1)} & =\frac{1}{2 \kappa^{2}} \int_{z=\infty} d^{d} x \sqrt{-\bar{\gamma}}\left(\bar{K}+\overline{\hat{n}}^{\mu} \bar{\nabla}_{\mu}\right) h, \\
S_{\mathrm{ct}}^{(1)} & =-\frac{d-1}{2 \kappa^{2} R} \int_{z=\infty} d^{d} x \sqrt{-\bar{\gamma}} h,
\end{aligned}
$$

and the second order boundary actions are

$$
\begin{aligned}
& S_{\mathrm{GH}}^{(2)}=\frac{1}{4 \kappa^{2}} \int_{z=\infty} d^{d} x \sqrt{-\bar{\gamma}}\left\{\left(\bar{K}+\overline{\hat{n}}^{\mu} \bar{\nabla}_{\mu}\right)\left(\frac{1}{2} h^{2}-h^{\mu \nu} h_{\mu \nu}\right)\right\}, \\
& S_{\mathrm{ct}}^{(2)}=-\frac{d-1}{4 \kappa^{2} R} \int_{z=\infty} d^{d} x \sqrt{-\bar{\gamma}}\left\{\frac{1}{2} h^{2}-h^{\mu \nu} h_{\mu \nu}\right\} .
\end{aligned}
$$

The background EOMs are

$$
\begin{aligned}
\overline{\mathcal{R}}_{\mu \nu}-\frac{1}{2} \overline{\mathcal{R}}_{\mu \nu}+\Lambda \bar{g}_{\mu \nu} & =\frac{1}{2}\left[\bar{F}_{\mu \rho}^{\mathbf{I}} \bar{F}_{\nu}^{\mathbf{I} \rho}-\frac{1}{4} \bar{g}_{\mu \nu}\left(\bar{F}^{\mathbf{I}}\right)^{2}\right], \\
\bar{\nabla}_{\mu} \bar{F}^{\mathbf{I} \mu \nu}+\lambda \epsilon^{\mathbf{I J K}} \bar{A}_{\mu}^{\mathbf{J}} \bar{F}^{\mathbf{K} \mu \nu} & =0
\end{aligned}
$$

and the linearized EOMs are

$$
\begin{aligned}
& {\left[\bar{\nabla}^{2}+\overline{\mathcal{R}}-2 \Lambda-\frac{1}{4}\left(\bar{F}^{\mathbf{I}}\right)^{2}\right] h_{\mu \nu}+\bar{\nabla}_{\mu} \bar{\nabla}_{\nu} h-\bar{\nabla}_{\rho} \bar{\nabla}_{(\mu} h_{\nu)}^{\rho}} \\
& =h^{\rho \sigma}\left(\bar{F}_{\mu \rho}^{\mathbf{I}} \bar{F}_{\nu \sigma}^{\mathbf{I}}-\frac{\bar{g}_{\mu \nu}}{d-1} \bar{F}_{\rho \eta}^{\mathbf{I}} \bar{F}_{\sigma}^{\mathbf{I} \eta}\right)+\bar{F}_{\rho(\mu}^{\mathbf{I}} F_{\nu)}^{\mathbf{I}(1) \rho}+\frac{1}{d-1} \bar{g}_{\mu \nu} \bar{F}^{\mathbf{I} \rho \sigma} F_{\rho \sigma}^{\mathbf{I}(1)}, \\
& \bar{\nabla}_{\mu}\left(F^{\mathbf{I}(1) \mu \nu}-\bar{F}_{\rho}^{\mathbf{I} \nu} h^{\mu \rho}\right)+\lambda \epsilon^{\mathbf{I J K}} \bar{A}_{\mu}^{\mathbf{J}}\left(F^{\mathbf{K}(1) \mu \nu}-\bar{F}_{\rho}^{\mathbf{K}{ }^{\nu} h^{\mu \rho}}\right) \\
& \quad+\bar{F}_{\rho}^{\mathbf{I} \mu} \bar{\nabla}_{\mu} h^{\nu \rho}+\frac{1}{2} \bar{F}^{\mathbf{I} \mu \nu} \bar{\nabla}_{\mu} h+\lambda \epsilon^{\mathbf{I J K}} a_{\mu}^{\mathbf{J}} \bar{F}^{\mathbf{K} \mu \nu}=0 .
\end{aligned}
$$

\section{Holographic $p_{x}+i p_{y}$ model}

\subsection{Background and its symmetries}

A general discussion on the AdS-black hole type solutions to the Einstein-SU(2) system can be found in [93]. Here we will only restrain to the simple model of [34]. We now go back to $d=3$ case and work in it for the rest of this paper. We choose the ansatz for the background to be

$$
\left\{\begin{array}{l}
d s^{2}=-F(z) d t^{2}+\frac{1}{F(z)} d z^{2}+r(z)^{2}\left(d x^{2}+d y^{2}\right) \\
\bar{A}_{t}^{\mathbf{3}}(z) \equiv \Phi(z), \quad \bar{A}_{x}^{\mathbf{1}}(z)=\bar{A}_{y}^{\mathbf{2}}(z) \equiv A(z)
\end{array}\right.
$$

and all other background gauge fields vanishing. $z=\infty$ is the boundary and $z=z_{H}$ is the horizon. When $A(z)=0$, the above background, and thus the ground state of the dual field theory, has two separate $\mathrm{U}(1)$ symmetries, one related to the rotation in $(x, y)$-plane 
and the other to the rotation in $(\mathbf{1}, \mathbf{2})$-vector space. The appearance of non-vanishing $A(z)$ breaks both symmetries, but preserves a combination of them - the U(1) symmetry of the joint rotations by the same angle in both $(x, y)$-plane and $(\mathbf{1}, \mathbf{2})$-vector space:

$$
\mathrm{U}(1)_{\theta}^{x y} \otimes \mathrm{U}(1)_{\phi}^{\mathbf{1 2}} \rightarrow \mathrm{U}(1)_{\theta=\phi}^{x y, \mathbf{1 2}}
$$

We can see that by introducing the non-vanishing $A(z)$ background, the originally separate symmetries in spacetime and $\mathrm{SU}(2)$ vector space are "locked" together. Similarly $A(z)$ breaks the separate parity symmetries in both spaces. When $\lambda=0$, i.e. when the $\mathrm{SU}(2)$ gauge field becomes a product of three $\mathrm{U}(1)$ fields, the joint parity symmetry in both spaces (reflection applied to both $(x, y)$-plane and $(\mathbf{1}, \mathbf{2})$-vector space simultaneously) is preserved:

$$
\mathbb{Z}_{2}^{x y} \otimes \mathbb{Z}_{2}^{12} \rightarrow \mathbb{Z}_{2}^{x y, 12}
$$

However, when $\lambda \neq 0$, the parity in $(\mathbf{1}, \mathbf{2})$-vector space is broken explicitly by the nonAbelian gauge connection $\lambda \epsilon^{\mathbf{I J K}} A_{\mu}^{\mathbf{J}} A_{\nu}^{\mathbf{K}}$ in the field strength, thus the parity in $(x, y)$-plane is also broken indirectly by this gauge connection term through the "locking" mechanism introduced by $A(z)$. Now we have a spacetime parity-breaking ground state thus the theory "appears" to be parity-broken and will have non-vanishing parity-violating transport coefficients such as Hall viscosity and Hall conductivity. In summary, to reach a spacetime parity-violating state, we first introduce a non-vanishing $A(z)$ to lock the spacetime symmetries and $\mathrm{SU}(2)$ vector space symmetries together, then break the $\mathrm{SU}(2)$ parity explicitly by making it non-Abelian, and this breaking will propagate to spatial parity.

The EOMs for background fields are

$$
\begin{aligned}
2 r(z)\left(\frac{d^{2}}{d z^{2}} r(z)\right)+\left(\frac{d}{d z} A(z)\right)^{2}+\frac{\lambda^{2} \Phi(z)^{2}}{F(z)^{2}} A(z)^{2} & =0, \\
\left(\frac{d^{2}}{d z^{2}} F(z)\right)-2 \frac{F(z)}{r(z)^{2}}\left(\frac{d}{d z} r(z)\right)^{2}-\left(\frac{d}{d z} \Phi(z)\right)^{2}-\frac{\lambda^{2}}{r(z)^{4}} A(z)^{4} & =0, \\
\frac{d}{d z}\left[r(z)^{2}\left(\frac{d}{d z} \Phi(z)\right)\right]-\frac{2 \lambda^{2} \Phi(z)}{F(z)} A(z)^{2} & =0, \\
\frac{d}{d z}\left[F(z)\left(\frac{d}{d z} A(z)\right)\right]+\lambda^{2}\left(\frac{\Phi(z)^{2}}{F(z)}-\frac{A(z)^{2}}{r(z)^{2}}\right) A(z) & =0,
\end{aligned}
$$

with a constraint equation derived from the trace of Einstein equation

$$
\left(\frac{d^{2}}{d z^{2}} F(z)\right)+4 \frac{F(z)}{r(z)}\left(\frac{d^{2}}{d z^{2}} r(z)\right)+2 \frac{F(z)}{r(z)^{2}}\left(\frac{d}{d z} r(z)\right)^{2}+\frac{4}{r(z)}\left(\frac{d}{d z} r(z)\right)\left(\frac{d}{d z} F(z)\right)=\frac{12}{R^{2}}
$$

Given that (4.2)-(4.5) are solved, (4.6) only fixes an integration constant (near boundary leading order coefficient of $F(z))$ in terms of the AdS radius $R$, thus it is not an independent differential equation, but rather an algebraic equation. This fact will play a role in later calculations. 


\subsection{Boundary conditions and thermodynamical functions}

The boundary conditions are imposed near the boundary $z=\infty$ by requiring the metric goes asymptotic AdS. Solving the above five equations near the boundary, we get

$$
\left\{\begin{array}{l}
r(z)=\frac{z}{R}+r_{1}+O\left(\frac{1}{z^{3}}\right) \\
F(z)=\left(\frac{z}{R}+r_{1}\right)^{2}+\frac{\Gamma}{z}+O\left(\frac{1}{z^{2}}\right) \\
\Phi(z)=\Phi_{0}+\frac{\Phi_{1}}{z}+O\left(\frac{1}{z^{2}}\right) \\
A(z)=\alpha_{0}+\frac{\alpha_{1}}{z}+O\left(\frac{1}{z^{2}}\right)
\end{array}\right.
$$

where $r_{1}, \Gamma, \Phi_{0}, \Phi_{1}, \alpha_{0}$ and $\alpha_{1}$ are constants. Two of them will be determined by two physical conditions to be discussed later, and the rest will be determined by the following (regularity) conditions near the horizon $z=z_{H}$ :

$$
\left\{\begin{array}{l}
r(z)=r\left(z_{H}\right)+O\left(z-z_{H}\right) \\
F(z)=4 \pi T\left(z-z_{H}\right)+O\left(\left(z-z_{H}\right)^{2}\right) \\
\Phi(z)=O\left(z-z_{H}\right) \\
A(z)=A\left(z_{H}\right)+O\left(z-z_{H}\right)
\end{array}\right.
$$

where $T$ is the Hawking temperature of the black hole and equals to the temperature of the field theory system on the boundary. The entropy density $s$, energy density $\varepsilon$, chemical potential $\mu$, charge density $\rho$ and order parameter $\langle\mathcal{O}\rangle$ can be expressed in the above asymptotic constants [34]:

$$
\begin{aligned}
s & =\frac{2 \pi}{\kappa^{2}} r\left(z_{H}\right)^{2}, \\
\varepsilon & =-\frac{\Gamma}{\kappa^{2} R^{2}}, \\
\mu & =\frac{\Phi_{0}}{2 R}, \\
\rho & =-\frac{\Phi_{1}}{\kappa^{2} R}, \\
\langle\mathcal{O}\rangle & =\frac{\alpha_{1}}{\kappa^{2} R},
\end{aligned}
$$

and $\alpha_{0}$ can be identified with an external source $J \sim \alpha_{0}$. Since we are looking for spontaneous symmetry breaking without an external source, the first physical (boundary) condition we impose is the vanishing of the source:

$$
\alpha_{0}=0 \text {. }
$$

The second physical condition is to fix either $\rho$ or $\mu$, depending on which ensemble one choose:

$$
\left\{\begin{array}{ll}
\rho=\text { constant } & \text { (Canonical Ensemble) } \\
\mu=\text { constant } & \text { (Grand Canonical Ensemble) }
\end{array} .\right.
$$


The characteristic function of the Canonical Ensemble - the Helmholtz free energy density $f_{\text {Helmholtz }}$ and that of the Grand Canonical Ensemble - the Grand Potential density $\Omega_{\text {Grand }}$ (equal to minus of the pressure) are

$$
\begin{aligned}
f_{\text {Helmholtz }} & =\varepsilon-T s, \\
\Omega_{\text {Grand }} & =f_{\text {Helmholtz }}-\mu \rho .
\end{aligned}
$$

\subsection{Background on-shell action}

The on-shell background bulk action is

$$
\bar{S}_{\text {bulk }}=\frac{1}{2 \kappa^{2}} \int d^{3} x \int_{z_{H}}^{\infty} d z\left\{\sqrt{-\bar{g}}\left[\overline{\mathcal{R}}-2 \Lambda-\frac{1}{4}\left(\bar{F}_{\mu \nu}^{\mathbf{I}}\right)^{2}\right]\right\} .
$$

By adding to the Lagrangian (the integrand inside " \{\} ") the following combination of background equations: $\frac{1}{2} r(z)^{2} \cdot[(4.3)+(4.6)]-A(z) \cdot(4.5)$, the integrand becomes a total derivative. Noticing the near horizon conditions (4.8), there is no contribution from the horizon. Thus we have

$$
\bar{S}_{\text {bulk }}=\frac{1}{2 \kappa^{2}} \int_{z=\infty} d^{3} x\left\{-F(z) \frac{d}{d z}\left(r(z)^{2}+\frac{1}{2} A(z)^{2}\right)\right\} .
$$

Together with the on-shell background boundary terms

$$
\bar{S}_{\mathrm{GH}}+\bar{S}_{\mathrm{ct}}=\frac{1}{2 \kappa^{2}} \int_{z=\infty} d^{3} x\left\{r(z)\left[r(z)\left(\frac{d}{d z} F(z)\right)+4 F(z)\left(\frac{d}{d z} r(z)\right)-\frac{4}{R} r(z)^{2} \sqrt{F(z)}\right]\right\}
$$

the total on-shell background action is

$$
\bar{S}=\frac{1}{2 \kappa^{2}} \int_{z=\infty} d^{3} x\left\{\frac{d}{d z}\left(r(z)^{2} F(z)\right)-F(z) A(z)\left(\frac{d}{d z} A(z)\right)-\frac{4}{R} r(z)^{2} \sqrt{F(z)}\right\} .
$$

Applying the boundary condition (4.7) it can be written as

$$
\bar{S}=\frac{1}{2 \kappa^{2}} \int_{z=\infty} d^{3} x \frac{-\Gamma+\alpha_{0} \alpha_{1}}{R^{2}} .
$$

The grand potential density equals to $-T$ multiplying the Euclidean on-shell action $(t$ is integrated from 0 to $\frac{1}{T}$ ) mod the volume:

$$
\Omega_{\text {Grand }}=-\frac{T}{V} \bar{S}_{\text {Euclidean }}=\frac{\Gamma-\alpha_{0} \alpha_{1}}{2 \kappa^{2} R^{2}}
$$

and by (4.17) the free energy density is

$$
f_{\text {Helmholtz }}=\frac{\Gamma-\Phi_{0} \Phi_{1}-\alpha_{0} \alpha_{1}}{2 \kappa^{2} R^{2}} .
$$

There is a useful identity for the background fields that can directly link the constants in near-boundary conditions (4.7) to those in near-horizon conditions (4.8). The combination of background equations $-F(z) \cdot(4.2)+r(z)^{2} \cdot(4.3)-\Phi(z) \cdot(4.4)-A(z) \cdot(4.5)$ is a total derivative, thus its integral is a constant:

$$
r(z)^{2} \frac{d}{d z}\left(F(z)-\frac{1}{2} \Phi(z)^{2}\right)-F(z) \frac{d}{d z}\left(r(z)^{2}+\frac{1}{2} A(z)^{2}\right)=\text { constant } .
$$


Evaluating it at both horizon and boundary using (4.8) and (4.7), we have

$$
4 \pi \operatorname{Tr}\left(z_{H}\right)^{2}=\frac{1}{R^{2}}\left(-3 \Gamma+\Phi_{0} \Phi_{1}+\alpha_{0} \alpha_{1}\right) .
$$

So through (4.26) one can see that the free energy density obtained above through the on-shell action and (4.17) is indeed the same as one can obtain directly from (4.16) by computing its right hand side.

\subsection{AdS-Reissner-Nordström solution}

One solution to equations (4.2)-(4.6) is the AdS-Reissner-Nordström (AdS-RN) solution:

$$
\left\{\begin{aligned}
r^{(0)}(z) & =\frac{z}{R} \\
F^{(0)}(z) & =\frac{z^{2}}{R^{2}}-\left(1+\frac{q^{2}}{4 \lambda^{2} R^{2}}\right) \frac{z_{H}^{3}}{R^{2} z}+\frac{q^{2} z_{H}^{4}}{4 \lambda^{2} R^{4} z^{2}} \\
\Phi^{(0)}(z) & =\frac{q z_{H}}{\lambda R^{2}}\left(1-\frac{z_{H}}{z}\right) \\
A^{(0)}(z) & =0
\end{aligned}\right.
$$

where $q$ is the dimensionless charge and it is related to the temperature $T$ and other parameters as

$$
T=\frac{3 z_{H}}{4 \pi R^{2}}\left(1-\frac{q^{2}}{12 \lambda^{2} R^{2}}\right) .
$$

The parameter $q$ and $T$ are confined between two limiting cases - the Schwarzschild limit

$$
\left\{\begin{aligned}
T & =\frac{3 z_{H}}{4 \pi R^{2}} \\
q & =0
\end{aligned}\right.
$$

and the Extremal limit

$$
\left\{\begin{array}{l}
T=0 \\
q=2 \sqrt{3} \lambda R
\end{array}\right.
$$

and as charge $q$ increases, the temperature $T$ decreases. The relation between $\mu, \rho$ and $T$ given by the AdS-Reissner-Nordström solution is

$$
\rho=\frac{4 \pi T \mu R^{2}}{3 \kappa^{2}}\left(1+\sqrt{1+\frac{3 \mu^{2}}{4 \pi^{2} T^{2}}}\right) .
$$

\subsection{Analytic approach to the symmetry-breaking solution}

When the temperature $T$ is below a certain critical temperature $T_{c}$ there exists another non-trivial solution to (4.2)-(4.6) that satisfies boundary conditions (4.7), (4.8), (4.14) and (4.15). This symmetry-breaking solution have been systematically discussed and numerically computed in [34]. Analytic approaches to solve similar models near the critical temperature in the Probe limit have also been studied in [94, 95]. The Probe limit is where the Yang-Mills coupling $\lambda R \gg 1$ while dimensionless charge $q$ is kept finite, or equivalently both chemical potential $\mu$ and charge density $\rho$ are very small compared to temperature 
$T$. In this limit, the back-reactions from the gauge fields to the metric is negligible at the leading order, so the background metric is an AdS-Schwarzschild black hole. But here, we will propose a more refined perturbative approach based on variational method to solve the system analytically for finite $\lambda R$ near the critical regime. The advantages of our approach are that it is a systematic method to go beyond the Probe limit and compute all the back-reactions between metric and gauge fields, and it is extremely suitable to be directly applied on the analytic computation of Hall viscosity and its ratio to angular momentum density, as will be shown in the next two sections.

Before proceeding to the actual calculation, we will first make a note here about how we will present our finite $\lambda R$ results in this paper. In our calculations we don't make any assumptions about how big or small $\lambda R$ is. But the expressions we obtain from the variational method for finite $\lambda R$ are usually very complicated and not illuminating. To present them in a better way, for every result we will make a large $\lambda R$ series expansion and keep only the first two leading terms. The leading terms are the same as one can get from the Probe limit; the next-to-leading terms can only be obtained by fully taking into account the back-reactions. The next-to-next-to-leading order terms are usually of order $O\left(\frac{1}{(\lambda R)^{4}}\right)$ compared to the leading terms. This means that even for, say $\lambda R=2$, the relative error due to the series expansion is just about $6 \%$. So it is reasonable to believe that results presented in this way are not only accurate for $\lambda R \gg 1$, but also very good down to $\lambda R \sim O(1)$. The regime where $\lambda R$ is really small and the above series expansion can not hold corresponds to the low temperature limit, and we will deal this regime separately in a late section at the end of this paper. Thus we will eventually get a qualitatively complete result for all possible range of $\lambda R$.

\subsection{Critical line and phase diagram}

The first step in our analytic approach is to find the expression for the critical temperature $T_{c}$ in terms of other physical parameters, i.e. the phase diagram.

To start, notice that at $T=T_{c}$ the symmetry-breaking solution with $A(z) \neq 0$ transits continuously to the AdS-Reissner-Nordström solution with $A(z)=0$. So when $T$ is below but very close to $T_{c}, A(z)$ is very small and can be treated perturbatively, thus the nonlinear equations (4.2)-(4.6) can be linearized and solved order by order. Let $\epsilon \ll 1$ be a book-keeping parameter of the perturbative expansion. It marks the "smallness" of $A(z)$ near the critical temperature, thus marks the deviation from the AdS-Reissner-Nordström solution (4.27) order by order, and at the end we will always set $\epsilon=1$. The ansatz for the background fields is:

$$
\left\{\begin{array}{l}
A(z)=A^{(1)}(z) \epsilon+A^{(2)}(z) \epsilon^{3}+O\left(\epsilon^{5}\right) \\
r(z)=r^{(0)}(z)+r^{(1)}(z) \epsilon^{2}+r^{(2)}(z) \epsilon^{4}+O\left(\epsilon^{6}\right) \\
F(z)=F^{(0)}(z)+F^{(1)}(z) \epsilon^{2}+F^{(2)}(z) \epsilon^{4}+O\left(\epsilon^{6}\right) \\
\Phi(z)=\Phi^{(0)}(z)+\Phi^{(1)}(z) \epsilon^{2}+\Phi^{(2)}(z) \epsilon^{4}+O\left(\epsilon^{6}\right)
\end{array} .\right.
$$


The first equation to solve is the linearized equation for $A^{(1)}(z)$ from (4.5):

$$
\frac{d}{d z}\left[F^{(0)}(z)\left(\frac{d}{d z} A^{(1)}(z)\right)\right]+\frac{\lambda^{2} \Phi^{(0)}(z)^{2}}{F^{(0)}(z)} A^{(1)}(z)=0 .
$$

Near horizon $z=z_{H}$, this equation has two characteristic solutions for $A^{(1)}(z)$ : one is regular and the other contains $\ln \left(z-z_{H}\right)$. We require $A^{(1)}(z)$ to be regular near horizon, thus choose one of the two integration constants to kill the $\ln \left(z-z_{H}\right)$ solution. Now for generic parameters this completely fixes the solution (particularly its near-boundary behavior at $z \rightarrow \infty$ ) up to an overall normalization constant. This means near the boundary $z \rightarrow \infty$ we have

$$
A^{(1)}(z)=\alpha_{0}^{(1)}+\frac{\alpha_{1}^{(1)}}{z}+O\left(\frac{1}{z^{2}}\right)
$$

where $\alpha_{0}^{(1)}$ being a fixed function of all parameters is generally non-vanishing. Thus generally the sourceless condition (4.14) can not be achieved only at linear level; its full realization requires the inclusion of higher order terms through the nonlinear term in (4.5), and this will also determine the behavior of the order parameter $\alpha_{1}^{(1)}$. However, there are special cases that the sourceless condition (4.14) can be achieved at linear level, when the parameters take some special discrete values. This corresponds to an eigenvalue problem for (4.33), and the eigensolution with the highest eigenvalue of $T$ (thus the lowest eigenvalue of $q$ ) corresponds precisely to the case $T=T_{c}$. So we have the condition for the critical temperature:

$$
\alpha_{0}^{(1)}\left(T=T_{c}, \lambda, \ldots\right)=0 .
$$

The above equation gives the expression for $T_{c}$ in terms of the other physical parameters $\lambda, \ldots$, thus the phase diagram.

Given the complicated form of (4.27), (4.33) can not be solved analytically in terms of special functions. We use variational method to solve it. Even though this is an approximate method, we will later see that its accuracy is surprisingly high. Solving (4.33) with appropriate boundary conditions corresponds to finding the extrema of the action

$$
I^{(1)}=\int_{z_{H}}^{\infty} d z\left[-F^{(0)}(z)\left(\frac{d}{d z} A^{(1)}(z)\right)^{2}+\frac{\lambda^{2} \Phi^{(0)}(z)^{2}}{F^{(0)}(z)} A^{(1)}(z)^{2}\right]+I_{\text {boundary }}^{(1)},
$$

where $I_{\text {boundary }}^{(1)}$ contains boundary terms such that under given boundary conditions the variational problem is well-defined (i.e. the variations of all boundary terms are vanishing). We have already imposed one regularity condition near the horizon. Given that the blackening function $F^{(0)}(z)$ is vanishing at the horizon, this condition does not introduce any boundary term to $I_{\text {boundary }}^{(1)}$. Now we fix the normalization of $A^{(1)}(z)$ by requiring that ${ }^{2}$

$$
\alpha_{1}^{(1)} \text { is fixed. }
$$

This introduces a boundary term

$$
I_{\text {boundary }}^{(1)}=-\frac{2 \alpha_{0}^{(1)} \alpha_{1}^{(1)}}{R^{2}} .
$$

\footnotetext{
${ }^{2}$ Notice that $\alpha_{1}$ is related to the order parameter $\langle\mathcal{O}\rangle$.
} 
Now we choose a form of the trial function: ${ }^{3}$

$$
A^{(1)}(z)=\alpha_{1}^{(1)}\left(1+\frac{z_{H}}{z}+\frac{z_{H}^{2}}{z^{2}}-\frac{q^{2} z_{H}^{3}}{4 \lambda^{2} R^{2} z^{3}}\right)\left(c_{0}^{(1)}+\frac{1-z_{H} c_{0}^{(1)}}{z}+\sum_{i=2}^{n} \frac{c_{i}^{(1)}}{z^{2}}\right)
$$

and then compute the action $I^{(1)}$ and solve $c_{i}^{(1)}$ by minimizing it:

$$
\frac{\partial I^{(1)}}{\partial c_{i}^{(1)}}=0 \quad(i=0,2,3, \ldots, n) .
$$

Notice that given the above trial ansatz,

$$
\alpha_{0}^{(1)}=c_{0}^{(1)} \alpha_{1}^{(1)}
$$

and the condition for critical temperature (4.35) becomes $c_{0}^{(1)}\left(T=T_{c}, \lambda, \ldots\right)=0$. We will not give the full expressions for $c_{i}^{(1)}$, since they are messy and interested readers can easily repeat the calculation. The numerator of $c_{0}^{(1)}$ is a bi-polynomial of $q$ and $\lambda R$ with high ranks, and finding the critical temperature according to the condition (4.35) corresponds to finding the roots of this polynomial: $q=q_{\text {root }}(\lambda R)$. However, even for the simplest ansatz the polynomial usually goes beyond rank-5 thus it does not have an explicit analytic expression for its roots. But numeric plot shows that these roots all have similar and simple behaviors in $(q, \lambda R)$-plane: they start at the origin, go closely along the extremal lines (4.30) and then at some points turn rapidly to constant- $q$ lines. Thus before the turning point, the solutions are essentially $T=0$. After the turning point, to obtain the constant- $q$ solutions, we can take the $\lambda R \gg 1$ limit in the numerator of $c_{0}^{(1)}$ and keeping only the first two leading terms will give good enough results. The actual critical temperature corresponds to the smallest $q_{\text {root }}$ :

$$
q_{c}=3.69-\frac{0.662}{(\lambda R)^{2}}+O\left(\frac{1}{(\lambda R)^{4}}\right) .
$$

To obtain the expression for critical temperature $T_{c}$, we need to use the ensemble conditions (4.15) to convert $z_{H}$ in (4.28) to physical parameters. For Canonical ensemble we have

$$
\frac{T_{c}}{\sqrt{\hat{\rho}}}=1.96 \sqrt{\lambda R}\left[1-\frac{1.04}{(\lambda R)^{2}}+O\left(\frac{1}{(\lambda R)^{4}}\right)\right] \theta\left(\lambda-\lambda_{c}\right),
$$

where $\hat{\rho}=\frac{\kappa^{2}}{(2 \pi)^{3} R^{2}} \rho$ as defined in [34]. For Grand Canonical ensemble we have

$$
\frac{T_{c}}{\mu}=0.129 \lambda R\left[1-\frac{0.954}{(\lambda R)^{2}}+O\left(\frac{1}{(\lambda R)^{4}}\right)\right] \theta\left(\lambda-\lambda_{c}\right)
$$

where $\theta(x)$ is the Heaviside step function. From the above two expressions we can see that $T_{c}$ will reach zero at a critical coupling around $\lambda_{c} R \approx 1$. We will deal with $T \rightarrow 0$

\footnotetext{
${ }^{3}$ The function in the first ( ) is to cancel the same factor in the blackening function $F^{(0)}(z)$ in the denominator of the action such that the integral is easy to do. One can of course choose other ansatz and will get similar results. $n$ is the rank of the trial polynomial. In practice one can only include the first few terms (like to set $n=4$ or 6 ) and will get very accurate results. We find that choosing $n$ to be an even number usually gives better results. In this paper, all calculations are done by setting $n=4$.
} 

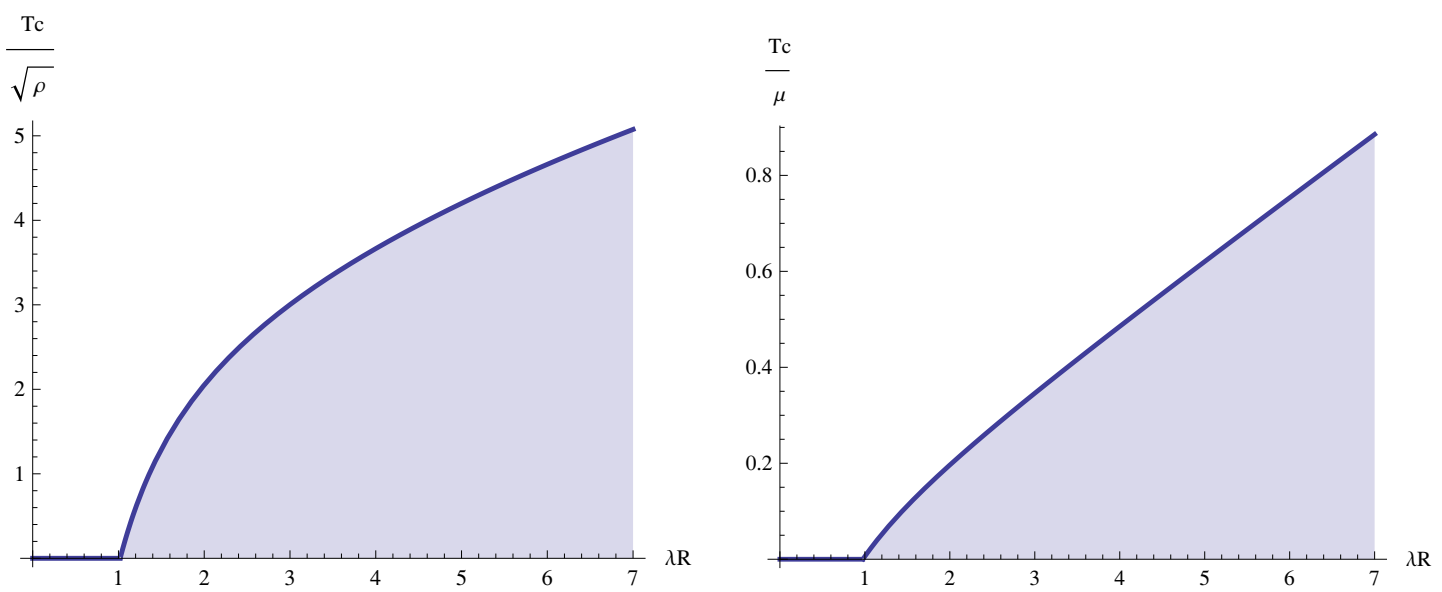

Figure 1. Phase diagrams for Canonical ensemble (left) and Grand Canonical ensemble (right). The blue lines are the critical lines given by equations (4.43) and (4.44). The shaded areas are the superconducting/superfluid phase and the unshaded areas the normal phase.

limit separately later to give a more accurate expression for this critical coupling $\lambda_{c}$. The phase diagrams are shown in figure 1 . Comparing with the numeric plot figure $1(\mathrm{~A})$ and equation (B8) in [34] we see quantitatively they are almost the same. Away from but close to the critical line (4.42) we find

$$
\begin{aligned}
c_{0}^{(1)} & =\frac{1}{\kappa R^{\frac{3}{2}} \sqrt{\rho \lambda}}\left[-2.10+\frac{8.31}{(\lambda R)^{2}}+O\left(\frac{1}{(\lambda R)^{4}}\right)\right]\left(1-\frac{T}{T_{c}}\right) \theta\left(T_{c}-T\right) \\
& =\frac{1}{\mu \lambda R^{3}}\left[-1.01+\frac{1.80}{(\lambda R)^{2}}+O\left(\frac{1}{(\lambda R)^{4}}\right)\right]\left(1-\frac{T}{T_{c}}\right) \theta\left(T_{c}-T\right),
\end{aligned}
$$

where the first line is for Canonical ensemble and second line Grand Canonical ensemble. This result will be very useful later.

\subsection{Near-critical behavior of order parameter}

Now going back to the boundary condition (4.35), we can see away from but close to $T_{c}$, we have

$$
\left.\alpha_{0}^{(1)}\right|_{T \rightarrow T_{c}}=-a^{(1)} \alpha_{1}^{(1)}\left(T_{c}-T\right),
$$

where $a^{(1)}$ is a positive quantity one can retrieve from (4.45). The fulfillment of the sourceless condition (4.14) now requires to include the next order solution:

$$
\alpha_{0}^{(1)}+\left.\alpha_{0}^{(2)}\right|_{T \rightarrow T_{c}}=0,
$$

where near the boundary

$$
A^{(2)}(z)=\alpha_{0}^{(2)}+\frac{\alpha_{1}^{(2)}}{z}+O\left(\frac{1}{z^{2}}\right)
$$

To proceed, we need to solve the other first order fields $r^{(1)}(z), F^{(1)}(z)$ and $\Phi^{(1)}(z)$ first. They satisfy a set of coupled second order inhomogeneous linear equations derived 
from (4.2)-(4.4), with all source terms quadratic in $A^{(1)}(z)$. These equations can be all put to integrable forms using the $O\left(\epsilon^{2}\right)$ order of the trace equation (4.6) and background equations which (4.27) satisfies, and then be integrated out one by one. The results are listed in appendix $\mathrm{C}$, with six arbitrary integration constants $C_{1}-C_{6}$ to be fixed by appropriate boundary conditions. $C_{1}$ is fixed by requiring that $r(z)$ goes asymptotically AdS near the boundary, that is, there is no $z$ term in $r^{(1)}(z)$ when $z \rightarrow \infty$. We also require $F(z) \rightarrow 4 \pi T\left(z-z_{H}\right)$ near the horizon, which means both the horizon position $z_{H}$ and the AdS-RN temperature-charge relation (4.28) are unchanged in the presence of the condensate $A^{(1)}(z)$. This implies $F^{(1)}(z) \rightarrow O\left(\left(z-z_{H}\right)^{2}\right)$, which fixes $C_{5}$ and $C_{6}$ in terms of the other constants through the vanishing of constant and linear terms near horizon. Notice that the asymptotic AdS requirement for $F^{(1)}(z)$ is automatically satisfied, thus fixes no more constant. The $O\left(\epsilon^{2}\right)$ order of the trace equation listed in appendix $\mathrm{C}$ fixes the constant $C_{2}$ in terms of the remaining. When solving this equation, one shall bear in mind that the equation (4.33) is not solved exactly, but just approximately by variational method. The consequence of that is that the $O\left(\epsilon^{2}\right)$ order trace equation will never be solved exactly either. To avoid this complication, we shall not require that the whole trace equation hold; instead, we will just look at the near boundary leading order of its left hand side and require this term alone to vanish. It is not hard to see that all the non-vanishing near boundary sub-leading orders we have omitted here are a consequence of (4.33) being solved approximately, and if (4.33) was solved exactly, they will all vanish automatically. Now we are left with two constants $C_{3}$ and $C_{4}$ to be fixed by conditions for $\Phi^{(1)}(z)$. One condition is obvious: we require $\Phi^{(1)}(z) \rightarrow O\left(z-z_{H}\right)$ near horizon such that $\Phi(z) \rightarrow O\left(z-z_{H}\right)$ in (4.8) holds. The vanishing of the near-horizon constant term fixes one of $C_{3}$ and $C_{4}$ in term of the other. By now, we have essentially exhausted all the boundary conditions we must impose for $r^{(1)}(z), F^{(1)}(z)$ and $\Phi^{(1)}(z)$ that are consequences of (4.7) and (4.8), but we are still left with an undetermined constant, basically a combination of $C_{3}$ and $C_{4}$. This is not surprising, because this is just a reflection of gauge freedoms related to $\Phi^{(1)}(z)$ and other fields at this order: the undetermined constant is associated with a pure-gauge solution, and this constant, if kept arbitrary, will not appear in any physical results that are expressed in terms of physical variables such as $T, \rho, \mu$ and $\lambda$ (note that $q$ and $z_{H}$ are not physical variables). We have explicitly verified this claim by keeping this constant arbitrary in all follow-up computations. But to make the computations more compact and transparent, we can use the gauge freedom to fix this arbitrary constant. There are two natural choices: if we choose to work in Canonical ensemble, in which $\rho \sim \Phi_{1}$ is kept fixed, we will hope that $\Phi_{1}$ is not altered in the presence of $A^{(1)}(z)$, thus we can require $\Phi^{(1)}(z) \rightarrow$ const. $+O\left(z^{-2}\right)$, i.e. $\Phi_{1}=\Phi_{1}^{(0)}$ and $\Phi_{1}^{(1)}=0$; on the other hand, if we choose to work in Grand Canonical ensemble, in which $\mu \sim \Phi_{0}$ is fixed, we can require $\Phi^{(1)}(z) \rightarrow O\left(z^{-1}\right)$, i.e. $\Phi_{0}=\Phi_{0}^{(0)}$ and $\Phi_{0}^{(1)}=0$, which means $\Phi_{0}$ is not altered in the presence of $A^{(1)}(z)$. Both choices, among others, shall give the same physical results at the end.

Since the actual calculation based on $A^{(1)}(z)$ obtained from variational method gives extremely baroque expressions for $r^{(1)}(z), \Phi^{(1)}(z)$ and $F^{(1)}(z)$ (as well as all second order fields to be discussed later), we will not give their explicit expressions in this paper. Inter- 
ested readers should be able to repeat our calculation easily following the procedures we have outlined here. We will only list some simple key results derived from them.

We are now at a position to solve second order fields. $A^{(2)}(z)$ satisfies

$$
\frac{d}{d z}\left[F^{(0)}(z)\left(\frac{d}{d z} A^{(2)}(z)\right)\right]+\frac{\lambda^{2} \Phi^{(0)}(z)^{2}}{F^{(0)}(z)} A^{(2)}(z)+\Upsilon^{(2)}(z)=0
$$

where

$$
\begin{aligned}
\Upsilon^{(2)}(z)= & \left(\frac{d}{d z} A^{(1)}(z)\right)\left[\left(\frac{d}{d z} F^{(1)}(z)\right)-\frac{F^{(1)}(z)}{F^{(0)}(z)}\left(\frac{d}{d z} F^{(0)}(z)\right)\right] \\
& -\lambda^{2} A^{(1)}(z)\left(\frac{A^{(1)}(z)^{2}}{r^{(0)}(z)^{2}}+2 \frac{\Phi^{(0)}(z)^{2} F^{(1)}(z)}{F^{(0)}(z)^{2}}-2 \frac{\Phi^{(0)}(z) \Phi^{(1)}(z)}{F^{(0)}(z)}\right)
\end{aligned}
$$

is a known source function in terms of the above solved first order fields. The action associated with the variational problem is

$I^{(2)}=\int_{z_{H}}^{\infty} d z\left[-F^{(0)}(z)\left(\frac{d}{d z} A^{(2)}(z)\right)^{2}+\frac{\lambda^{2} \Phi^{(0)}(z)^{2}}{F^{(0)}(z)} A^{(2)}(z)^{2}+2 \Upsilon^{(2)}(z) A^{(2)}(z)\right]+I_{\text {boundary }}^{(2)}$.

Since $A^{(2)}(z)$ satisfies an inhomogeneous equation with a source $\Upsilon^{(2)}(z)$, we can impose two boundary conditions to completely determine $A^{(2)}(z)$ in term of $\Upsilon^{(2)}(z)$ : one is again the regularity condition near the horizon, and the second is chosen to be

$$
\alpha_{1}^{(2)}=0
$$

where $\alpha_{1}^{(2)}$ is defined in (4.48). Actually in the perturbative expansion of $A(z)$, the splitting between $A^{(1)}(z)$ and $A^{(2)}(z)$ is arbitrary: one can always take a small part of $A^{(1)}(z)$ which is of the same order of $A^{(2)}(z)$ and sneak it into the latter and the perturbative expansion still holds. The meaning of the above boundary condition is just to make the splitting unique, or equivalently one can think it is the definition of $A^{(1)}(z)$. The above boundary condition introduces no more boundary term since itself is vanishing, thus

$$
I_{\text {boundary }}^{(2)}=0 \text {. }
$$

Using a trial ansatz

$$
A^{(2)}(z)=\left(1+\frac{z_{H}}{z}+\frac{z_{H}^{2}}{z^{2}}-\frac{q^{2} z_{H}^{3}}{4 \lambda^{2} R^{2} z^{3}}\right)\left(\alpha_{0}^{(2)}-\frac{z_{H} \alpha_{0}^{(2)}}{z}+\sum_{i=2}^{n} \frac{c_{i}^{(2)}}{z^{2}}\right)
$$

we can solve $\alpha_{0}^{(2)}$ and $c_{i}^{(2)}$ in a similar fashion as before. Since the source $\Upsilon^{(2)}(z)$ is cubic in $A^{(1)}(z)$ thus cubic in $\alpha_{1}^{(1)}$, it's not hard to see that close to $T_{c}$

$$
\left.\alpha_{0}^{(2)}\right|_{T \rightarrow T_{c}}=a^{(2)}\left(\alpha_{1}^{(1)}\right)^{3} .
$$


$a^{(2)}$ is a positive quantity in terms of other parameters:

$$
\begin{aligned}
a^{(2)} & =\frac{1}{\kappa^{5} R^{\frac{7}{2}} \rho^{\frac{5}{2}} \sqrt{\lambda}}\left[1.44-\frac{3.98}{(\lambda R)^{2}}+O\left(\frac{1}{(\lambda R)^{4}}\right)\right] \\
& =\frac{1}{R^{11} \mu^{5} \lambda^{3}}\left[0.688-\frac{0.783}{(\lambda R)^{2}}+O\left(\frac{1}{(\lambda R)^{4}}\right)\right],
\end{aligned}
$$

where the first line is for Canonical ensemble and the second line Grand Canonical ensemble. Now the sourceless condition (4.47) reads

$$
-a^{(1)} \alpha_{1}^{(1)}\left(T_{c}-T\right)+a^{(2)}\left(\alpha_{1}^{(1)}\right)^{3}=0 .
$$

Since both $a^{(1)}$ and $a^{(2)}$ are positive, ${ }^{4}$ when $T>T_{c}$ there is only one trivial solution $\alpha_{1}^{(1)}=0$, which means $A(z)=0$. This reproduces the AdS-RN solution and shows it is the only possible solution when $T>T_{c}$. When $T<T_{c}$ there is another non-trivial solution

$$
\alpha_{1}^{(1)}=\left(\frac{a^{(1)}}{a^{(2)}}\right)^{\frac{1}{2}} \sqrt{T_{c}-T} \quad\left(T<T_{c}\right),
$$

which yields a non-vanishing $A(z)$. Notice that $\alpha_{1}$ is proportional to the order parameter $\langle\mathcal{O}\rangle$, we obtain $\langle\mathcal{O}\rangle \sim \sqrt{T_{c}-T} \theta\left(T_{c}-T\right)$, which agrees with Ginzburg-Landau theory. For Canonical ensemble, we get ${ }^{5}$

$$
\frac{\langle\mathcal{O}\rangle}{\rho}=1.21\left[1-\frac{0.594}{(\lambda R)^{2}}+O\left(\frac{1}{(\lambda R)^{4}}\right)\right]\left(1-\frac{T}{T_{c}}\right)^{\frac{1}{2}} \theta\left(T_{c}-T\right) .
$$

For Grand Canonical ensemble

$$
\frac{\langle\hat{\mathcal{O}}\rangle}{\mu^{2}}=4.88 \times 10^{-3} \lambda R\left[1-\frac{0.322}{(\lambda R)^{2}}+O\left(\frac{1}{(\lambda R)^{4}}\right)\right]\left(1-\frac{T}{T_{c}}\right)^{\frac{1}{2}} \theta\left(T_{c}-T\right)
$$

where $\langle\hat{\mathcal{O}}\rangle=\frac{\kappa^{2}}{(2 \pi)^{3} R^{2}}\langle\mathcal{O}\rangle$ as defined in [34].

\subsection{Near-critical behaviors of charge density and chemical potential}

We can also compute the change of chemical potential $\mu$ or charge density $\rho$, when the other one is fixed, as a function of $T_{c}-T$ near the critical temperature. There are two contributions to it. The first one is from the AdS-RN part $\Phi^{(0)}$, which we can obtain by directly vary (4.31), and it is proportional to $T_{c}-T$. The second contribution is from $\Phi^{(1)}$,

\footnotetext{
${ }^{4}$ This assertion for $a^{(2)}$ need to be made carefully, and we will have more discussion on this later for low temperature limit.

${ }^{5}$ Compare to the numerical result in [36]: converting their equation (16) to our conventions,and noticing their definition for $\langle J\rangle$ involves a factor of $\sqrt{2}$, they have
}

$$
\frac{\langle\mathcal{O}\rangle}{\rho}=1.16\left(1-\frac{T}{T_{c}}\right)^{\frac{1}{2}} \theta\left(T_{c}-T\right)
$$

at the leading order. So the agreement is good. 
by computing either $\Phi_{1}^{(1)}$ for $\rho$ or $\Phi_{0}^{(1)}$ for $\mu$, which is proportional to $\langle\mathcal{O}\rangle^{2}$, thus also to $T_{c}-T$. So we can see that they are of the same order. For Canonical ensemble, we get

$$
\mu-\mu_{c}=0.165 \sqrt{\frac{\rho}{\lambda}} \frac{\kappa}{R^{\frac{3}{2}}}\left[1-\frac{1.63}{(\lambda R)^{2}}+O\left(\frac{1}{(\lambda R)^{4}}\right)\right]\left(1-\frac{T}{T_{c}}\right) \theta\left(T_{c}-T\right)
$$

where

$$
\mu_{c}(\rho, \lambda)=0.960 \sqrt{\frac{\rho}{\lambda}} \frac{\kappa}{R^{\frac{3}{2}}}\left[1-\frac{0.0898}{(\lambda R)^{2}}+O\left(\frac{1}{(\lambda R)^{4}}\right)\right] .
$$

For Grand Canonical ensemble we get

$$
\rho-\rho_{c}=-0.318 \lambda \mu^{2} \frac{R^{3}}{\kappa^{2}}\left[1-\frac{1.04}{(\lambda R)^{2}}+O\left(\frac{1}{(\lambda R)^{4}}\right)\right]\left(1-\frac{T}{T_{c}}\right) \theta\left(T_{c}-T\right),
$$

where $\rho_{c}(\mu, \lambda)$ can be obtained by inverting the above expression of $\mu_{c}(\rho, \lambda)$.

Now we can rewrite $\langle\mathcal{O}\rangle$ in terms of $\mu-\mu_{c}$ and $\rho-\rho_{c}$ :

$$
\begin{aligned}
\langle\mathcal{O}\rangle & =2.98 \lambda^{\frac{1}{4}} \rho^{\frac{3}{4}} \frac{R^{\frac{3}{4}}}{\sqrt{\kappa}}\left[1+\frac{0.220}{(\lambda R)^{2}}+O\left(\frac{1}{(\lambda R)^{4}}\right)\right] \sqrt{\mu-\mu_{c}} \theta\left(\mu-\mu_{c}\right) \\
& =2.15 \sqrt{\lambda} \mu \frac{R^{\frac{3}{2}}}{\kappa}\left[1+\frac{0.197}{(\lambda R)^{2}}+O\left(\frac{1}{(\lambda R)^{4}}\right)\right] \sqrt{\rho_{c}-\rho} \theta\left(\rho_{c}-\rho\right),
\end{aligned}
$$

where the first line is for Canonical ensemble and second line Grand Canonical ensemble. The critical line has

$$
\mu-\mu_{c}=\frac{\partial \mu_{c}}{\partial \rho}\left(\rho-\rho_{c}\right)=\frac{0.480}{\sqrt{\lambda \rho}}\left[1-\frac{0.0898}{(\lambda R)^{2}}+O\left(\frac{1}{(\lambda R)^{4}}\right)\right] \frac{\kappa}{R^{\frac{3}{2}}}\left(\rho-\rho_{c}\right) .
$$

It is straightforward to check that if the calculation is consistent, then the ratio of the first line over the second line in the above expression of $\langle\mathcal{O}\rangle$ shall be 1 if we substitute in the absolute value of (4.65). We get

$$
1.00+\frac{0.067}{(\lambda R)^{2}}+O\left(\frac{1}{(\lambda R)^{4}}\right) .
$$

We believe the $\lambda R$ term is a numeric error because the variational method is approximate. So within this error our calculation for the two ensembles is consistent.

\subsection{Near-critical behaviors of characteristic functions}

Next it is straightforward to solve other second order fields $r^{(2)}(z), F^{(2)}(z)$ and $\Phi^{(2)}(z)$ following the same procedures for first order fields as discussed before, then use (4.24) and (4.23) to compute characteristic functions for the two ensembles. For Canonical ensemble, we find the free energy density of the unbroken phase (pure AdS-RN background) is

$$
f_{\text {Helmholtz }}^{(0)}=-\frac{32 \pi^{3} R^{2}}{27 \kappa^{2}} T^{3}+\frac{3 \kappa^{2}}{8 \pi R^{2}} \frac{\rho^{2}}{T}+O\left(\frac{1}{\lambda^{2}}\right)
$$

and the free energy density difference between the broken phase $(A(z) \neq 0)$ and unbroken phase near the critical temperature is

$$
\Delta f_{\text {Helmholtz }}=-\frac{6.45}{\lambda R} \rho T_{c}\left(1-\frac{T}{T_{c}}\right)^{2} \theta\left(T_{c}-T\right)+O\left(\frac{1}{\lambda^{3}}\right),
$$


while for Grand Canonical ensemble the grand potential density of the unbroken phase is

$$
\Omega_{\text {Grand }}^{(0)}=-\frac{32 \pi^{3} R^{2}}{27 \kappa^{2}} T^{3}+O\left(\frac{1}{\lambda^{2}}\right)
$$

and the grand potential density difference between the broken and unbroken phase near the critical temperature is

$$
\Delta \hat{\Omega}_{\mathrm{Grand}}=-1.61 \times 10^{-3} \lambda R \mu^{3}\left(1-\frac{T}{T_{c}}\right)^{2} \theta\left(T_{c}-T\right)+O\left(\frac{1}{\lambda}\right),
$$

where $\hat{\Omega}_{\text {Grand }}=\frac{\kappa^{2}}{(2 \pi)^{3} R^{2}} \Omega_{\text {Grand }}$. Notice that in both cases, below $T_{c}$ the broken phase has lower free energy (grand potential) density than the unbroken phase, so it is the preferred phase and the phase transition can indeed happen; and the fact that the characteristic functions are quadratic in $T_{c}-T$ indicates that this is a second order phase transition.

\section{Tensor mode fluctuations and viscosities}

\subsection{Mode classification}

We now look at metric and gauge field fluctuations in the background (4.1). Their EOMs are given by (3.16) and (3.17). We will work in momentum space where coordinates $(t, x, y)$ are Fourier transformed to momentum $\left(\omega, k_{x}, k_{y}\right)$. All fluctuations in (3.16) and (3.17) are highly coupled with each others, mainly because the joint $\mathrm{U}(1)$ symmetry in $(x, y)$ plane and $(\mathbf{1}, \mathbf{2})$-vector space is now broken explicitly by any non-zero spatial momentum vector $\vec{k}=\left(k_{x}, k_{y}\right) \neq 0$. In this case the usual classification of tensor, vector (shear) and scalar (sound) modes are not very helpful because they do not decouple from each other. However, since we are only interested in viscosities, which are only related to $\omega$ terms in the correlation functions, we can assume the fluctuations to be spatially homogeneous, i.e. $\vec{k}=0$. Now the joint $\mathrm{U}(1)$ symmetry is respected and we find there are indeed three decoupled modes, which behave as tensor, vector and scalar under the joint rotation of both $(x, y)$-plane and $(\mathbf{1}, \mathbf{2})$-vector space by the same angle:

- Tensor mode: $\left\{h_{x y}, a_{y}^{\mathbf{1}}+a_{x}^{\mathbf{2}}\right\},\left[h_{x x}-h_{y y}, a_{x}^{\mathbf{1}}-a_{y}^{\mathbf{2}}\right]$;

- Vector mode: $\left\{h_{t x}+h_{t y}, h_{x z}+h_{y z}, a_{t}^{\mathbf{1}}+a_{t}^{\mathbf{2}}, a_{z}^{\mathbf{1}}+a_{z}^{2}, a_{x}^{\mathbf{3}}+a_{y}^{\mathbf{3}}\right\},\left[h_{t x}-h_{t y}, h_{x z}-h_{y z}\right.$, $\left.a_{t}^{1}-a_{t}^{2}, a_{z}^{1}-a_{z}^{2}, a_{x}^{\mathbf{3}}-a_{y}^{\mathbf{3}}\right]$

- Scalar mode: $h_{t t}, h_{z z}, h_{z t}, h_{x x}+h_{y y}, a_{t}^{3}, a_{z}^{3}, a_{x}^{1}+a_{y}^{2}, a_{y}^{1}-a_{x}^{2}$.

The EOM for each combination of fields listed above is just the same combination of corresponding components of (3.16) or (3.17). Notice that above in both tensor and vector mode we group the fields into two brackets: the ones in " \{\} " are all even under the joint parity operation - the simultaneously exchange of $x \leftrightarrow y$ and $\mathbf{1} \leftrightarrow \mathbf{2}$, while those in "[ ]" are all odd. When this joint parity is a symmetry of the system, the fields in each bracket do not mix with those in the other bracket, thus inside each of tensor and vector modes there are two decoupled sub-modes which are eigenstates of the joint parity. However, here the non-Abelian coupling $\lambda$ explicitly breaks the joint parity, so fields in two brackets do mix, and one can expect that the mixing terms must all proportional to $\lambda$. 


\subsection{Tensor mode EOMs}

We now focus on tensor mode fluctuations, through which we will obtain viscosities. For concreteness, we define shorthand notations

$$
\begin{array}{rlrl}
h_{x y} & \equiv r(z)^{2} h_{e}(\omega, z), & & \frac{1}{2}\left(a_{y}^{\mathbf{1}}+a_{x}^{\mathbf{2}}\right) \equiv a_{e}(\omega, z), \\
\frac{1}{2}\left(h_{x x}-h_{y y}\right) & \equiv r(z)^{2} h_{o}(\omega, z), & \frac{1}{2}\left(a_{x}^{\mathbf{1}}-a_{y}^{\mathbf{2}}\right) \equiv a_{o}(\omega, z),
\end{array}
$$

where the subscript $e$ or $o$ means whether that field is even or odd under the joint parity operation. Using background equations (4.2)-(4.6), the equations that the above fields satisfy can be put into the following forms:

$$
\begin{aligned}
\frac{d}{d z}\left[r(z)^{2} F(z)\left(\frac{d}{d z} h_{i}(\omega, z)\right)\right] & =S_{i}^{h}(\omega, z ; \lambda), \\
\frac{d}{d z}\left[F(z)\left(\frac{d}{d z} a_{i}(\omega, z)\right)\right]+\frac{\lambda^{2} \Phi(z)^{2}}{F(z)} a_{i}(\omega, z) & =S_{i}^{a}(\omega, z ; \lambda),
\end{aligned}
$$

where $i, j=e, o,{ }^{6}$ and the sources are

$$
\begin{aligned}
S_{i}^{h}(\omega, z ; \lambda)= & -\left[\omega^{2} \frac{r(z)^{2}}{F(z)}+\frac{\lambda^{2} \Phi(z)^{2}}{F(z)} A(z)^{2}-F(z)\left(\frac{d}{d z} A(z)\right)^{2}\right] h_{i}(\omega, z) \\
& +2\left[-F(z)\left(\frac{d}{d z} A(z)\right)\left(\frac{d}{d z} a_{i}(\omega, z)\right)+\frac{\lambda^{2} \Phi(z)^{2}}{F(z)} A(z) a_{i}(\omega, z)\right] \\
& +2 i \omega \frac{\lambda \Phi(z)}{F(z)} A(z) \epsilon_{i j} a_{j}(\omega, z), \\
S_{i}^{a}(\omega, z ; \lambda)= & {\left[F(z)\left(\frac{d}{d z} A(z)\right)\left(\frac{d}{d z} h_{i}(\omega, z)\right)+\frac{\lambda^{2}}{r(z)^{2}} A(z)^{3} h_{i}(\omega, z)\right] } \\
& -\left(\frac{\omega^{2}}{F(z)}+\frac{\lambda^{2}}{r(z)^{2}} A(z)^{2}\right) a_{i}(\omega, z) \\
& +i \omega \frac{\lambda \Phi(z)}{F(z)} \epsilon_{i j}\left[A(z) h_{j}(\omega, z)-2 a_{j}(\omega, z)\right] .
\end{aligned}
$$

The totally antisymmetric "tensor" $\epsilon_{i j}$ is defined as $\epsilon_{e o}=-\epsilon_{o e}=1, \epsilon_{e e}=\epsilon_{o o}=0$ and the repeated subscript $j$ is summed over $e$ and $o$. Since we are only interested in the hydrodynamic regime where momentum is small compared to other scales $(\omega \ll 2 \pi T)$, we can treat $\omega$ perturbatively. Furthermore, we focus on near-critical behaviors of the transport coefficients, so we can treat $A(z)$ perturbatively as well. Noticing that every term in $S_{i}^{h}(\omega, z ; \lambda)$ and $S_{i}^{a}(\omega, z ; \lambda)$ is proportional to either $\omega$ or $A(z)$, all sources can be treated perturbatively. We will expand all fields $h_{i}(\omega, z)$ and $a_{i}(\omega, z)$ as double series of $\omega$ and $A(z)$ and (5.3) and (5.4) can be solved order by order by just integrating the sources. It's straightforward to integrate (5.3), but for (5.4) it's not that obvious because the appearance of $a_{i}(\omega, z)$ term on the left hand side spoils the apparent integrability. To integrate (5.4), we need to use its Green's functions (bulk-to-bulk propagators in the context of holography), which are worked out in appendix A.

\footnotetext{
${ }^{6}$ In this section we will reserve the letters $i$ and $j$ exclusively for $e$ and $o$.
} 


\subsection{Boundary-to-bulk propagators}

First let us solve the indicial equations of (5.3) and (5.4) near the boundary and horizon to get the solution's asymptotic behaviors. Using (4.8) in (5.3) and (5.4) we get

$$
h_{i}(\omega, z), a_{i}(\omega, z) \rightarrow\left(z-z_{H}\right)^{ \pm i \frac{\omega}{4 \pi T}} \quad\left(z \rightarrow z_{H}\right)
$$

and using (4.7) we get

$$
\left\{\begin{array}{l}
h_{i}(\omega, z) \rightarrow 1 \text { or } z^{-3} \\
a_{i}(\omega, z) \rightarrow 1 \text { or } z^{-1}
\end{array} \quad(z \rightarrow \infty) .\right.
$$

We turn on only the $h_{i}$ boundary fields $\bar{h}_{i}$ since we are only interested in calculating the energy-stress tensor correlators, so the boundary condition we impose is:

$$
\left\{\begin{array}{l}
h_{i}(\omega, z) \rightarrow \bar{h}_{i} \\
a_{i}(\omega, z) \rightarrow 0
\end{array} \quad(z \rightarrow \infty) .\right.
$$

Following [87-89] we choose the incoming wave condition near the horizon:

$$
h_{i}(\omega, z), a_{i}(\omega, z) \rightarrow\left(z-z_{H}\right)^{-i \frac{\omega}{4 \pi T}} \quad\left(z \rightarrow z_{H}\right) .
$$

Following appendix A, let us assume that $\Theta_{m}(z)(m=<,>)$ are the two independent solutions to the homogeneous equation associated with (5.4):

$$
\frac{d}{d z}\left[F(z)\left(\frac{d}{d z} \Theta_{m}(z)\right)\right]+\frac{\lambda^{2} \Phi(z)^{2}}{F(z)} \Theta_{m}(z)=0
$$

with the boundary condition

$$
\left\{\begin{array}{ll}
\Theta_{<}(z) \text { is regular } & \left(z \rightarrow z_{H}\right) \\
\Theta_{>}(z) \rightarrow O\left(z^{-1}\right) & (z \rightarrow \infty)
\end{array} .\right.
$$

Notice that given the above boundary condition, usually $\Theta_{<}(z) \rightarrow$ constant when $z \rightarrow \infty$. Assume

$$
\left\{\begin{array}{l}
\Theta_{<}(z)=B_{<}+O\left(\frac{1}{z}\right) \\
\Theta_{>}(z)=\frac{B_{>}}{z}+O\left(\frac{1}{z^{2}}\right) \quad(z \rightarrow \infty),
\end{array}\right.
$$

then the normalization constant is

$$
N_{r}=F(z) \operatorname{Wr}\left[\Theta_{<}(z), \Theta_{>}(z)\right]=-\frac{B_{<} B_{>}}{R^{2}} .
$$

Now we list the solution to (5.3) and (5.4) as a double series expansion of small $\omega$ and $A(z)$ up to orders $O(\omega)$ and $O\left(A(z)^{4}\right)$ :

$$
\begin{aligned}
& h_{i}(\omega, z)=\left(\frac{z-z_{H}}{z}\right)^{-i \frac{\omega}{4 \pi T}}\left\{\bar{h}_{i}+\sum_{j=1}^{4} h_{i}^{(0 j)}(z)+i \omega\left(\sum_{j=0}^{4} h_{i}^{(1 j)}(z)\right)+O\left(\omega^{2}, A(z)^{5}\right)\right\}, \\
& a_{i}(\omega, z)=\left(\frac{z-z_{H}}{z}\right)^{-i \frac{\omega}{4 \pi T}}\left\{\sum_{j=0}^{4} a_{i}^{(0 j)}(z)+i \omega\left(\sum_{j=0}^{4} a_{i}^{(1 j)}(z)\right)+O\left(\omega^{2}, A(z)^{5}\right)\right\},
\end{aligned}
$$


where each term carries two superscripts: the first one labels order in $\omega$ and the second one order in $A(z)$. Many of them are vanishing and we list below only the non-vanishing ones:

$$
\begin{aligned}
& h_{i}^{(02)}(z)=\bar{h}_{i} \int_{\infty}^{z} d \xi \frac{A(\xi)}{r(\xi)^{2}}\left(\frac{d}{d \xi} A(\xi)\right), \\
& a_{i}^{(03)}(z)=\bar{h}_{i} \frac{1}{N_{r}}\left\{-\Theta_{<}(z) \int_{\infty}^{z} d \xi \Theta_{>}(\xi) \frac{A(\xi)}{r(\xi)^{2}}\left[F(\xi)\left(\frac{d}{d \xi} A(\xi)\right)^{2}+\lambda^{2} A(\xi)^{2}\right]\right. \\
& \left.+\Theta_{>}(z) \int_{z_{H}}^{z} d \xi \Theta_{<}(\xi) \frac{A(\xi)}{r(\xi)^{2}}\left[F(\xi)\left(\frac{d}{d \xi} A(\xi)\right)^{2}+\lambda^{2} A(\xi)^{2}\right]\right\}, \\
& h_{i}^{(04)}(z)=\bar{h}_{i} h^{(04)}(z) \text {, } \\
& h_{i}^{(10)}(z)=\bar{h}_{i}\left\{\frac{1}{4 \pi T} \ln \left(\frac{z-z_{H}}{z}\right)-r\left(z_{H}\right)^{2} \int_{\infty}^{z} d \xi \frac{1}{r(\xi)^{2} F(\xi)}\right\}, \\
& a_{i}^{(11)}(z)=\bar{h}_{i} \frac{r\left(z_{H}\right)^{2}}{N_{r}}\left\{\Theta_{<}(z) \int_{\infty}^{z} d \xi \Theta_{>}(\xi) \frac{1}{r(\xi)^{2}}\left(\frac{d}{d \xi} A(\xi)\right)\right. \\
& \left.-\Theta_{>}(z) \int_{z_{H}}^{z} d \xi \Theta_{<}(\xi) \frac{1}{r(\xi)^{2}}\left(\frac{d}{d \xi} A(\xi)\right)\right\} \\
& +\epsilon_{i j} \bar{h}_{j} \frac{\lambda}{N_{r}}\left\{\Theta_{>}(z) \int_{z_{H}}^{z} d \xi \Theta_{<}(\xi) \frac{\Phi(\xi) A(\xi)}{F(\xi)}-\Theta_{<}(z) \int_{\infty}^{z} d \xi \Theta_{>}(\xi) \frac{\Phi(\xi) A(\xi)}{F(\xi)}\right\}, \\
& h_{i}^{(12)}(z)=\bar{h}_{i}\left\{\left[\frac{1}{4 \pi T} \ln \left(\frac{z-z_{H}}{z}\right)-r\left(z_{H}\right)^{2} \int_{\infty}^{z} d \rho \frac{1}{r(\rho)^{2} F(\rho)}\right] \int_{\infty}^{z} d \xi \frac{A(\xi)}{r(\xi)^{2}}\left(\frac{d}{d \xi} A(\xi)\right)\right. \\
& +2 r\left(z_{H}\right)^{2} \int_{\infty}^{z} d \rho \frac{1}{r(\rho)^{2} F(\rho)} \int_{z_{H}}^{\rho} d \xi \frac{A(\xi)}{r(\xi)^{2}}\left(\frac{d}{d \xi} A(\xi)\right) \\
& +2 \frac{r\left(z_{H}\right)^{2}}{N_{r}}\left[\int_{\infty}^{z} d \rho \frac{\Theta_{>}(\rho)}{r(\rho)^{2}}\left(\frac{d}{d \rho} A(\rho)\right) \int_{z_{H}}^{\rho} d \xi \frac{\Theta_{<}(\xi)}{r(\xi)^{2}}\left(\frac{d}{d \xi} A(\xi)\right)\right. \\
& \left.\left.-\int_{\infty}^{z} d \rho \frac{\Theta_{<}(\rho)}{r(\rho)^{2}}\left(\frac{d}{d \rho} A(\rho)\right) \int_{\infty}^{\rho} d \xi \frac{\Theta_{>}(\xi)}{r(\xi)^{2}}\left(\frac{d}{d \xi} A(\xi)\right)\right]\right\} \\
& +\epsilon_{i j} \bar{h}_{j} \frac{2 \lambda}{N_{r}}\left\{\int_{\infty}^{z} d \rho \frac{\Theta_{<}(\rho)}{r(\rho)^{2}}\left(\frac{d}{d \rho} A(\rho)\right) \int_{\infty}^{\rho} d \xi \frac{\Theta_{>}(\xi) \Phi(\xi) A(\xi)}{F(\xi)}\right. \\
& \left.-\int_{\infty}^{z} d \rho \frac{\Theta_{>}(\rho)}{r(\rho)^{2}}\left(\frac{d}{d \rho} A(\rho)\right) \int_{z_{H}}^{\rho} d \xi \frac{\Theta_{<}(\xi) \Phi(\xi) A(\xi)}{F(\xi)}\right\}, \\
& a_{i}^{(13)}(z)=\bar{h}_{i} a^{(13)}(z) \\
& +\epsilon_{i j} \bar{h}_{j} \frac{\lambda}{N_{r}}\left\{\Theta_{>}(z) \int_{z_{H}}^{z} d \xi \Theta_{<}(\xi) S^{(13)}(\xi)-\Theta_{<}(z) \int_{\infty}^{z} d \xi \Theta_{>}(\xi) S^{(13)}(\xi)\right\}, \\
& h_{i}^{(14)}(z)=\bar{h}_{i} h^{(14)}(z)+\epsilon_{i j} \bar{h}_{j} \lambda\left\{\int_{\infty}^{z} d \rho \frac{1}{r(\rho)^{2} F(\rho)}\left[\int_{z_{H}}^{\rho} d \xi S_{\text {Hall }}(\xi)+S_{\text {null }}(\rho)\right]\right\}
\end{aligned}
$$


with

$$
\begin{aligned}
S^{(13)}(\xi)= & \frac{\Phi(\xi) A(\xi)}{F(\xi)} \int_{\infty}^{\xi} d \rho \frac{A(\rho)}{r(\rho)^{2}}\left(\frac{d}{d \rho} A(\rho)\right)+\frac{1}{N_{r} r(\xi)^{2}}\left[2 F(\xi)\left(\frac{d}{d \xi} A(\xi)\right)^{2}+\lambda^{2} A(\xi)^{2}\right] \\
& \times\left(\Theta_{<}(\xi) \int_{\infty}^{\xi} d \rho \Theta>(\rho) \frac{\Phi(\rho) A(\rho)}{F(\rho)}-\Theta_{>}(\xi) \int_{z_{H}}^{\xi} d \rho \Theta_{<}(\rho) \frac{\Phi(\rho) A(\rho)}{F(\rho)}\right) \\
& +\frac{2 \Phi(\xi)}{N_{r} F(\xi)}\left\{\Theta_{<}(\xi) \int_{\infty}^{\xi} d \rho \Theta>(\rho) \frac{A(\rho)}{r(\rho)^{2}}\left[F(\rho)\left(\frac{d}{d \rho} A(\rho)\right)^{2}+\lambda^{2} A(\rho)^{2}\right]\right. \\
& \left.-\Theta_{>}(\xi) \int_{z_{H}}^{\xi} d \rho \Theta<(\rho) \frac{A(\rho)}{r(\rho)^{2}}\left[F(\rho)\left(\frac{d}{d \rho} A(\rho)\right)^{2}+\lambda^{2} A(\rho)^{2}\right]\right\} \\
S_{\text {null }}(\rho)= & F(\rho)\left(\frac{d}{d \rho} A(\rho)\right)\left\{\frac { 2 } { N _ { r } } \left(\Theta<(\rho) \int_{\infty}^{\rho} d \xi \Theta(\xi) S^{(13)}(\xi)\right.\right. \\
& \left.\left.-\Theta_{>}(\rho) \int_{z_{H}}^{\rho} d \xi \Theta<(\xi) S^{(13)}(\xi)\right)+A(\rho) S_{1}^{(14)}(\rho)\right\}+S_{2}^{(14)}(\rho), \\
S_{1}^{(14)}(\rho)= & \frac{2}{N_{r}}\left\{\int_{\infty}^{\rho} d \xi \frac{\Theta<(\xi)}{r(\xi)^{2}}\left(\frac{d}{d \xi} A(\xi)\right) \int_{\infty}^{\xi} d \sigma \frac{\Theta>(\sigma) \Phi(\sigma) A(\sigma)}{F(\sigma)}\right. \\
& \left.-\int_{\infty}^{\rho} d \xi \frac{\Theta>(\xi)}{r(\xi)^{2}}\left(\frac{d}{d \xi} A(\xi)\right) \int_{z_{H}}^{\xi} d \sigma \frac{\Theta<(\sigma) \Phi(\sigma) A(\sigma)}{F(\sigma)}\right\}, \\
& \left.-\int_{\infty}^{\rho} d \xi \frac{\Theta_{>}(\xi) \Phi(\xi) A(\xi)}{F(\xi)} \int_{z_{H}}^{\rho} d \sigma \frac{\Theta<(\sigma) A(\sigma) F(\sigma)}{r(\sigma)^{2}}\left(\frac{d}{d \sigma} A(\sigma)\right)^{2}\right\} \\
S_{2}^{(14)}(\rho)= & \int_{z_{H}}^{\rho} d \xi \frac{\Theta<(\xi) \Phi(\xi) A(\xi)}{F(\xi)} \int_{\infty}^{\rho} d \sigma \frac{\Theta>(\sigma) A(\sigma) F(\sigma)}{r(\sigma)^{2}}\left(\frac{d}{d \sigma} A(\sigma)\right)^{2}
\end{aligned}
$$

and most importantly

$$
\begin{aligned}
S_{\text {Hall }}(\xi)= & \frac{2}{N_{r}} \frac{\Phi(\xi) A(\xi)}{F(\xi)}\left\{\Theta_{>}(\xi) \int_{z_{H}}^{\xi} d \sigma \Theta_{<}(\sigma) \frac{A(\sigma)}{r(\sigma)^{2}}\left[2 F(\sigma)\left(\frac{d}{d \sigma} A(\sigma)\right)^{2}+\lambda^{2} A(\sigma)^{2}\right]\right. \\
& \left.-\Theta_{<}(\xi) \int_{\infty}^{\xi} d \sigma \Theta_{>}(\sigma) \frac{A(\sigma)}{r(\sigma)^{2}}\left[2 F(\sigma)\left(\frac{d}{d \sigma} A(\sigma)\right)^{2}+\lambda^{2} A(\sigma)^{2}\right]\right\}
\end{aligned}
$$

Our final results of correlators up to the desired order will not contain $h^{(04)}(z), h^{(14)}(z)$ and $a^{(13)}(z)$, so we will not give their explicit expressions here. Notice that $S_{\text {null }}(z) \rightarrow 0$ near the boundary $z \rightarrow \infty$. Because of this, we will see later that $S_{\text {null }}(z)$ will drop off in the final expression for Hall viscosity.

\subsection{2-point functions and viscosities}

We follow the prescriptions in [87-89] to obtain causal 2-point functions from the above bulk-to-boundary propagators and the second order on-shell action (3.8). The total on-shell 
boundary action for the tensor mode is

$$
\begin{aligned}
S^{(2)}= & \frac{1}{2 \kappa^{2}} \int_{z=\infty} d^{3} x \sum_{i=e, o}\left\{-\frac{1}{2} r(z)^{2} F(z)\left[h_{i}\left(\frac{d}{d z} h_{i}\right)\right]-F(z)\left[a_{i}\left(\frac{d}{d z} a_{i}\right)\right]\right. \\
& \left.-\frac{1}{2}\left[\frac{d}{d z}\left(r(z)^{2} F(z)\right)-\frac{4}{R} r(z)^{2} \sqrt{F(z)}\right] h_{i}^{2}+F(z)\left(\frac{d}{d z} A(z)\right) h_{i} a_{i}\right\} .
\end{aligned}
$$

For a causal 2-point function, one of the two operators has earlier time than the other one. The causal prescription is that in every term in the above action, substitute one of the two fluctuation fields with the advanced boundary-to-bulk propagator, whose boundary fields are identified as the sources to the earlier-time operators, and substitute the other fluctuation field with the retarded boundary-to-bulk propagator, whose boundary fields are identified as sources to the later-time operators. ${ }^{7}$ The solution we obtain in the previous section using the incoming-wave condition near the horizon, (5.15) and (5.16), are the advanced boundary-to-bulk propagators. Notice that the equations (5.3) and (5.4) are invariant under the simultaneous reflections of $\omega \rightarrow-\omega$ and $\lambda \rightarrow-\lambda$, the retarded boundary-to-bulk propagators can be obtained from the advanced ones also by these operations. The readers should pay attention to the operation $\lambda \rightarrow-\lambda$, which is very crucial for getting the correct results. By taking functional derivatives of the substituted boundary action (5.30) with respect to the boundary fields $\bar{h}_{i}$, we get the causal 2-point functions: ${ }^{8}$

$$
\begin{aligned}
G_{\mathrm{ra}}^{x x-y y, x y}(\omega) & =-i \omega\left(\frac{\lambda}{\kappa^{2}} \int_{z_{H}}^{\infty} d z S_{\mathrm{Hall}}(z)\right)+O\left(\omega^{2}, A(z)^{6}\right) \\
G_{\mathrm{ra}}^{x y, x y}(\omega) & =-\frac{\Gamma}{2 \kappa^{2} R^{2}}-i \omega \frac{r\left(z_{H}\right)^{2}}{2 \kappa^{2}}\left[1-2 \int_{z_{H}}^{\infty} d z \frac{A(z)}{r(z)^{2}}\left(\frac{d}{d z} A(z)\right)\right]+O\left(\omega^{2}, A(z)^{4}\right),
\end{aligned}
$$

where $\Gamma$ is defined in (4.7). Compare with Kubo formulae

$$
\begin{aligned}
G_{\mathrm{ra}}^{x x-y y, x y}(\omega) & =-2 i \eta_{H} \omega+O\left(\omega^{2}\right), \\
G_{\mathrm{ra}}^{x y, x y}(\omega) & =p-i \eta \omega+O\left(\omega^{2}\right),
\end{aligned}
$$

where $p\left(=\frac{1}{2} \varepsilon\right), \eta$ and $\eta_{H}$ are the pressure, shear viscosity and Hall viscosity of the system, and also notice the entropy density $s$ given by (4.9), we have

$$
\eta_{H}=\frac{\lambda}{2 \kappa^{2}} \int_{z_{H}}^{\infty} d z S_{\text {Hall }}(z)+O\left(A(z)^{6}\right)
$$

and

$$
\frac{\eta}{s}=\frac{1}{4 \pi}\left\{1-2 \int_{z_{H}}^{\infty} d z \frac{A(z)}{r(z)^{2}}\left(\frac{d}{d z} A(z)\right)+O\left(A(z)^{4}\right)\right\}
$$

\footnotetext{
${ }^{7}$ Since every term is quadratic in fluctuations, there are two permutations of substitution for each term.

${ }^{8}$ The other two 2-point functions one can compute are not independent from the above ones:
}

$$
G_{\mathrm{ra}}^{x y, x x-y y}(\omega)=G_{\mathrm{ra}}^{x x-y y, x y}(-\omega), \quad G_{\mathrm{ra}}^{x x-y y, x x-y y}(\omega)=4 G_{\mathrm{ra}}^{x y, x y}(\omega) .
$$


where $S_{\text {Hall }}(z)$ is given by (5.29). Notice that close to $T_{c}, A(z)$ is monotonic and vanishes at $z=\infty$, which means the second term in the above ratio of $\eta / s$ is always positive, thus the universal lower bound of $1 / 4 \pi$ for $\eta / s$ is not violated up to this order. This is the question [96] tried to address but failed, and we provide the answer now. For Canonical ensemble, we get

$$
\frac{\eta}{s}=\frac{1}{4 \pi}\left\{1+\frac{0.821}{(\lambda R)^{2}}\left[1-\frac{0.672}{(\lambda R)^{2}}+O\left(\frac{1}{(\lambda R)^{4}}\right)\right]\left(\frac{T_{c}-T}{T_{c}}\right) \theta\left(T_{c}-T\right)\right\} .
$$

For Grand Canonical ensemble

$$
\frac{\eta}{s}=\frac{1}{4 \pi}\left\{1+\frac{0.701}{(\lambda R)^{2}}\left[1-\frac{0.486}{(\lambda R)^{2}}+O\left(\frac{1}{(\lambda R)^{4}}\right)\right]\left(\frac{T_{c}-T}{T_{c}}\right) \theta\left(T_{c}-T\right)\right\} .
$$

This ratio for anisotropic holographic $p$-wave superfluid models has been computed in [97-99] and the behavior they found is similar to our result.

\subsection{Bulk-to-bulk propagators}

Now we will solve the two unknown functions $\Theta_{<}(z)$ and $\Theta_{>}(z)$ in the bulk-to-bulk propagators near the critical temperature and obtain a more compact formula for Hall viscosity. Notice that (5.11) is the same as (4.5) except for the non-linear $A(z)^{3}$ term. So we will use the same variational method to solve $\Theta(z)$ here, as we did in section 4 for $A(z)$.

Let us start with $\Theta_{<}(z)$. First, we make a perturbative expansion:

$$
\Theta_{<}(z)=\Theta_{<}^{(1)}(z) \epsilon+\Theta_{<}^{(2)}(z) \epsilon^{3}+O\left(\epsilon^{5}\right)
$$

and its near boundary value in (5.13) will get a similar expansion:

$$
B_{<}=B_{<}^{(1)} \epsilon+B_{<}^{(2)} \epsilon^{3}+O\left(\epsilon^{5}\right) .
$$

The function $F(z)$ and $\Phi(z)$ in (5.11) have already been expanded in (4.32) and computed in section 4 , so we can just cite the results there. For the first order, the EOM that $\Theta_{<}^{(1)}(z)$ satisfies is exactly the same as (4.33), and we also impose the same near-horizon regularity condition for them, so we can choose

$$
\Theta_{<}^{(1)}(z)=A^{(1)}(z),
$$

then

$$
B_{<}^{(1)}=\alpha_{0}^{(1)}=c_{0}^{(1)} \alpha_{1}^{(1)},
$$

where $c_{0}^{(1)}$ is given in (4.45) and $\alpha_{1}^{(1)} \propto\langle\mathcal{O}\rangle$. Next we will solve for $\Theta_{<}^{(2)}$, similarly as we did for $A^{(2)}(z)$, but not exactly the same, since their equations differ by the non-linear $A^{(1)}(z)^{3}$ term. From now on we will define

$$
\Theta_{<}^{(2)} \equiv \tilde{A}^{(2)}(z)
$$

The EOM for $\tilde{A}^{(2)}(z)$ is

$$
\frac{d}{d z}\left[F^{(0)}(z)\left(\frac{d}{d z} \tilde{A}^{(2)}(z)\right)\right]+\frac{\lambda^{2} \Phi^{(0)}(z)^{2}}{F^{(0)}(z)} \tilde{A}^{(2)}(z)+\tilde{\Upsilon}^{(2)}(z)=0
$$


where

$$
\begin{aligned}
\tilde{\Upsilon}^{(2)}(z)= & \left(\frac{d}{d z} A^{(1)}(z)\right)\left[\left(\frac{d}{d z} F^{(1)}(z)\right)-\frac{F^{(1)}(z)}{F^{(0)}(z)}\left(\frac{d}{d z} F^{(0)}(z)\right)\right] \\
& -2 \lambda^{2} A^{(1)}(z) \frac{\Phi^{(0)}(z)}{F^{(0)}(z)^{2}}\left(\Phi^{(0)}(z) F^{(1)}(z)-F^{(0)}(z) \Phi^{(1)}(z)\right) .
\end{aligned}
$$

Comparing the above expression with $\Upsilon^{(2)}(z)$ in section 4 , the only difference is the $A^{(1)}(z)^{3}$ term. The action associated with the variational problem is

$$
\tilde{I}^{(2)}=\int_{z_{H}}^{\infty} d z\left[-F^{(0)}(z)\left(\frac{d}{d z} \tilde{A}^{(2)}(z)\right)^{2}+\frac{\lambda^{2} \Phi^{(0)}(z)^{2}}{F^{(0)}(z)} \tilde{A}^{(2)}(z)^{2}+2 \tilde{\Upsilon}^{(2)}(z) \tilde{A}^{(2)}(z)\right]+\tilde{I}_{\text {boundary }}^{(2)} .
$$

Following section 4, we impose the same boundary conditions for $\tilde{A}^{(2)}(z)$ : one is the regularity condition near the horizon, and the other is

$$
\tilde{\alpha}_{1}^{(2)}=0,
$$

where $\tilde{\alpha}_{1}^{(2)}$ is defined in the ${ }^{\sim}$ version of (4.48). We also have

$$
\tilde{I}_{\text {boundary }}^{(2)}=0 \text {. }
$$

The trial ansatz is

$$
\tilde{A}^{(2)}(z)=\left(1+\frac{z_{H}}{z}+\frac{z_{H}^{2}}{z^{2}}-\frac{q^{2} z_{H}^{3}}{4 \lambda^{2} R^{2} z^{3}}\right)\left(\tilde{\alpha}_{0}^{(2)}-\frac{z_{H} \tilde{\alpha}_{0}^{(2)}}{z}+\sum_{i=2}^{n} \frac{\tilde{c}_{i}^{(2)}}{z^{2}}\right)
$$

and we can solve $\tilde{\alpha}_{0}^{(2)}$ and $\tilde{c}_{i}^{(2)}$ in a similar fashion as before. We get $\tilde{\alpha}_{0}^{(2)} \propto\left(\alpha_{1}^{(1)}\right)^{3}$. Define $\tilde{\alpha}_{0}^{(2)}=\tilde{c}_{0}^{(2)} \alpha_{1}^{(1)}$, then $\tilde{c}_{0}^{(2)} \propto\left(\alpha_{1}^{(1)}\right)^{2} \propto\langle\mathcal{O}\rangle^{2}$. We then have

$$
B_{<}^{(2)}=\tilde{\alpha}_{0}^{(2)}=\tilde{c}_{0}^{(2)} \alpha_{1}^{(1)}
$$

and

$$
B_{<}=B_{<}^{(1)}+B_{<}^{(2)}=\alpha_{0}^{(1)}+\tilde{\alpha}_{0}^{(2)}=\left(c_{0}^{(1)}+\tilde{c}_{0}^{(2)}\right) \alpha_{1}^{(1)} .
$$

Notice that near the critical line, both $c_{0}^{(1)} \propto \tilde{c}_{0}^{(2)} \propto\left(T_{c}-T\right)$, so $B_{<}^{(1)}$ are in fact of the same order as $B_{<}^{(2)}$ ! The calculation gives

$$
\begin{aligned}
c_{0}^{(1)}+\tilde{c}_{0}^{(2)} & =\frac{1}{\kappa R^{\frac{3}{2}} \sqrt{\rho \lambda}}\left[-0.603+\frac{0.366}{(\lambda R)^{2}}+O\left(\frac{1}{(\lambda R)^{4}}\right)\right]\left(1-\frac{T}{T_{c}}\right) \theta\left(T_{c}-T\right) \\
& =\frac{1}{\mu \lambda R^{3}}\left[-0.495+\frac{0.253}{(\lambda R)^{2}}+O\left(\frac{1}{(\lambda R)^{4}}\right)\right]\left(1-\frac{T}{T_{c}}\right) \theta\left(T_{c}-T\right),
\end{aligned}
$$

where the first line is for Canonical ensemble and second line Grand Canonical ensemble. Notice that with the cubic term $A(z)^{3}$ in place, we will get $\alpha_{0}^{(1)}+\alpha_{0}^{(2)}=c_{0}^{(1)}+c_{0}^{(2)}=0$, which is exactly how the sourceless condition (4.47) is realized. Another comment we would like to 
make is regarding the gauge freedom associated with $\Phi^{(1)}(z)$ which is discussed in section 4: only the combination $c_{0}^{(1)}+\tilde{c}_{0}^{(2)}$ is gauge-invariant (i.e. independent of the coefficient of that pure gauge solution); both $c_{0}^{(1)}$ and $\tilde{c}_{0}^{(2)}$ alone are not gauge-invariant when written in terms of physical variables like $T$ near the critical line.

Next we will solve $\Theta_{>}(z)$, but this is much easier. Using (5.14), we have the Wronskian $W_{r}\left[\Theta_{<}(z), \Theta_{>}(z)\right] \propto B_{<} \propto c_{0}^{(1)}+\tilde{c}_{0}^{(2)} \propto T_{c}-T$, which vanishes at $T=T_{c}$. Thus at the critical line, $\Theta_{<}(z)$ and $\Theta_{>}(z)$ are not linearly independent: they are just proportional to each other. Thus at the leading order, we can choose $\Theta_{>}^{(1)}(z)=\Theta_{<}^{(1)}(z)=A^{(1)}(z)$. From now on for simplicity we will omit the superscript "(1)" in $A^{(1)}(z)$ when there is no confusion, since throughout this paper we always talk about near critical regime. Thus we have

$$
\Theta_{>}(z)=\Theta_{<}(z)=A(z)
$$

From (5.13) we have

$$
B_{>}=\alpha_{1}^{(1)}
$$

then from (5.14) the normalization constant of the bulk Green's function is

$$
N_{r}=-\frac{1}{R^{2}}\left(c_{0}^{(1)}+\tilde{c}_{0}^{(2)}\right)\left(\alpha_{1}^{(1)}\right)^{2}
$$

where $c_{0}^{(1)}+\tilde{c}_{0}^{(2)}$ is given in $(5.50)$.

\subsection{Hall viscosity}

Now the two terms in (5.29) can be combined and then factored out, which gives a simpler expression for Hall viscosity near the critical temperature:

$$
\eta_{H}=\left(\frac{\lambda}{\kappa^{2}} \int_{z_{H}}^{\infty} d \xi \frac{\Phi(\xi) A(\xi)^{2}}{F(\xi)}\right)\left\{\frac{1}{N_{r}} \int_{z_{H}}^{\infty} d \sigma \frac{A(\sigma)^{2}}{r(\sigma)^{2}}\left[2 F(\sigma)\left(\frac{d}{d \sigma} A(\sigma)\right)^{2}+\lambda^{2} A(\sigma)^{2}\right]\right\} .
$$

We can see the above expression is factorized into two parts. To compute the above expression, we set $A(z)=A^{(1)}(z), r(z)=r^{(0)}(z), F(z)=F^{(0)}(z), \Phi(z)=\Phi^{(0)}(z)$ and use the analytic solutions obtained via variational method and $N_{r}$ given by (5.52). For both ensembles, the second complicated factor inside " \{\} " turns out to be

$$
1.02-\frac{0.025}{(\lambda R)^{2}}+O\left(\frac{1}{(\lambda R)^{4}}\right)
$$

along the critical line, which is always close to 1 , since the sub-leading orders give negligible corrections, even for small $\lambda R$. Finally, for Canonical ensemble, we have

$$
\begin{aligned}
\frac{\eta_{H}}{\rho} & =\frac{1.09}{\lambda R}\left[1-\frac{0.68}{(\lambda R)^{2}}+O\left(\frac{1}{(\lambda R)^{4}}\right)\right]\left(1-\frac{T}{T_{c}}\right) \theta\left(T_{c}-T\right), \\
\frac{\eta_{H}}{s} & =\frac{0.639}{(\lambda R)^{2}}\left[1-\frac{0.86}{(\lambda R)^{2}}+O\left(\frac{1}{(\lambda R)^{4}}\right)\right]\left(1-\frac{T}{T_{c}}\right) \theta\left(T_{c}-T\right) .
\end{aligned}
$$


For Grand Canonical ensemble, we have

$$
\begin{aligned}
& \frac{\eta_{H}}{\mu^{2}}=1.01\left[1-\frac{0.32}{(\lambda R)^{2}}+O\left(\frac{1}{(\lambda R)^{4}}\right)\right] \frac{R^{2}}{\kappa^{2}}\left(1-\frac{T}{T_{c}}\right) \theta\left(T_{c}-T\right) \\
& \frac{\eta_{H}}{s}=\frac{0.545}{(\lambda R)^{2}}\left[1-\frac{0.67}{(\lambda R)^{2}}+O\left(\frac{1}{(\lambda R)^{4}}\right)\right]\left(1-\frac{T}{T_{c}}\right) \theta\left(T_{c}-T\right) .
\end{aligned}
$$

\section{Vector mode fluctuations and angular momentum}

\subsection{Edge current and angular momentum density}

In this section we only study the static case, so there is no $t$-dependence anywhere, and $\omega=0$. For fluids in $(2+1)$-dimensional flat Minkowskian space, the $t$-component of the equation of energy-stress tensor conservation reads

$$
\partial_{i} T^{t i}(\vec{x})=0
$$

and has the solution

$$
T^{t i}(\vec{x})=\epsilon^{i j} \partial_{j} \vartheta(\vec{x}),
$$

where $i, j, k=x, y, \vartheta(\vec{x})$ is an arbitrary function and the totally anti-symmetric tensor $\epsilon^{i j}$ in flat 2-dimensional Euclidean space is normalized to $\epsilon^{x y}=1$. To proceed, we put the fluid in a box of size $b$ (the shape does not really matter, even though we assume it is a square) and at the end we can send $b \rightarrow \infty$. If the fluid is almost homogeneous in the box, then to the leading order in derivative expansion, we have

$$
\vartheta(\vec{x})= \begin{cases}\frac{1}{2} \ell & \left(|x| \leqslant \frac{1}{2} b,|y| \leqslant \frac{1}{2} b\right) \\ 0 & \text { (otherwise) }\end{cases}
$$

where $\ell$ is a constant. So we can see $T^{t i}(\vec{x})$ is vanishing both inside and outside the fluid (box), and is only non-vanishing at the boundary of the fluid:

$$
T^{t i}(\vec{x})=\frac{1}{2} \ell \epsilon^{i j}\left[-\delta\left(x^{j}-\frac{1}{2} b\right)+\delta\left(x^{i}+\frac{1}{2} b\right)\right] \theta\left(\frac{1}{2} b-|x|\right) \theta\left(\frac{1}{2} b-|y|\right) .
$$

This is a momentum flow around the edge of the fluid - the edge current, and $\ell$ characterizes its strength. The direction of the edge current is always along the edge, either clockwise or counter-clockwise, depending on the sign of $\ell$. Notice that here for the uniform hydrodynamic limit, $T^{t i}(\vec{x})$ at the leading order is vanishing everywhere inside the bulk of the fluid, and this "boundary" nature must be related to the topological nature of the underlying field theory, as is commonly recognized in the study of Hall effect and other parity-breaking effects. This "boundary" nature, also appearing in its holographic dual theory, requires us to handle the boundary terms with extreme care, as will be shown in the next subsection. There were previous reports about failures of finding angular momenta which were expected to be non-vanishing, and we suspect that the mishandling of boundary terms could be a potential cause of the failures. In this section we will show how to handle the boundary terms correctly to get expected results. 
Since $T^{t i}$ is the momentum density, the total angular momentum $L$ of the fluid can be defined in the usual way:

$$
L=\int d^{2} \vec{x} \epsilon_{i j} x^{i} T^{t j}(\vec{x}) .
$$

Use (6.2), (6.3) and $\epsilon_{i j} \epsilon^{j k}=-\delta_{i}^{k}$, then integrate by parts, we have

$$
L=\int d^{2} \vec{x} \vartheta(\vec{x}) \partial_{i} x^{i}=\ell \int_{|x|,|y| \leqslant \frac{1}{2} b} d^{2} \vec{x}=\ell V_{2},
$$

where $V_{2}=b^{2}$ is the volume of the fluid (box). We can see that $\ell$ is the angular momentum density of the fluid.

\subsection{Linear on-shell action}

On the other hand, the 1st order on-shell action linear to metric fluctuations is the source term to the energy-stress tensor:

$$
S^{(1)}=\frac{1}{2} \int d^{3} x \bar{h}_{\mu \nu}(x) T^{\mu \nu}(x) .
$$

For the static case when we turn on only $\bar{h}_{t i}(\vec{x})$ sources and use the above results, we have

$$
S^{(1)}=\int d^{3} x \bar{h}_{t i}(\vec{x}) T^{t i}(\vec{x})=\int d^{3} x \bar{h}_{t i}(\vec{x}) \epsilon^{i j} \partial_{j} \vartheta(\vec{x}) .
$$

Integrate by parts and let $b \rightarrow \infty$, we have:

$$
S^{(1)}=\frac{\ell}{2} \int d^{3} x \epsilon^{i j} \partial_{i} \bar{h}_{t j}(\vec{x}) .
$$

It is well known that in holography $S^{(1)}$ is an integral of total derivatives, as shown above, and thus a boundary term, because background EOMs make the bulk part vanish. The usual treatment is to say that the boundary terms are vanishing at "boundaries" like $x, y=\frac{1}{2} b$ and only non-vanishing at the AdS boundary $z=\infty$. However, here due to the edge current effect, we will not assume the boundary terms vanishing at $x, y=\frac{1}{2} b$. Actually once we have obtained $S^{(1)}$ in the bulk, we will not integrate out the total derivatives; instead we will just keep it as a bulk integral over total derivatives, and by comparing it with (6.9) we can read off $\ell$ directly from its coefficient. Notice that in (6.9) we have ignored higher order derivative terms since we assume $\vartheta(\vec{x})$ is almost homogeneous inside the fluid, so it is essentially a hydrodynamic expansion up to leading order in derivatives.

Now we calculate $S^{(1)}=S_{\mathrm{bulk}}^{(1)}+S_{\mathrm{GH}}^{(1)}+S_{\mathrm{ct}}^{(1)}$ from the bulk action (3.7) and its associated boundary terms in the background (4.1). There are four different parts in $S^{(1)}$. The first part is the $z$-derivative term in (3.7), which is a boundary term at the AdS boundary $z=\infty$, together with the two other boundary terms $S_{\mathrm{GH}}^{(1)}$ and $S_{\mathrm{ct}}^{(1)}$. Its contribution is

$$
\begin{aligned}
\frac{1}{2 \kappa^{2}} \int_{z=\infty} & d^{3} x\left\{2 r(z)\left[F(z)\left(\frac{d}{d z} r(z)\right)-\frac{r(z)}{R} \sqrt{F(z)}\right] h_{t}^{t}(\vec{x}, z)\right. \\
& +\frac{1}{2}\left[\frac{d}{d z}\left(r(z)^{2} F(z)\right)-\frac{4}{R} r(z)^{2} \sqrt{F(z)}\right]\left[h_{x}^{x}(\vec{x}, z)+h_{y}^{y}(\vec{x}, z)\right] \\
& \left.+r(z)^{2}\left(\frac{d}{d z} \Phi(z)\right) a_{t}^{\mathbf{3}}(\vec{x}, z)-F(z)\left(\frac{d}{d z} A(z)\right)\left[a_{x}^{\mathbf{1}}(\vec{x}, z)+a_{y}^{\mathbf{2}}(\vec{x}, z)\right]\right\} .
\end{aligned}
$$


Using (4.7) this is

$$
\frac{1}{2 \kappa^{2}} \int_{z=\infty} d^{3} x\left\{\frac{\Gamma}{2 R^{2}}\left[2 \bar{h}_{t}^{t}(\vec{x})-\bar{h}_{x}^{x}(\vec{x})-\bar{h}_{y}^{y}(\vec{x})\right]-\frac{\Phi_{1}}{R^{2}} \bar{a}_{t}^{\mathbf{3}}(\vec{x})+\frac{\alpha_{1}}{R^{2}}\left[\bar{a}_{x}^{1}(\vec{x})+\bar{a}_{y}^{2}(\vec{x})\right]\right\} .
$$

Since we only turn on $\bar{h}_{t x}(\vec{x})$ and $\bar{h}_{t y}(\vec{x})$ boundary fields for angular momentum, the above term has no contribution to $S^{(1)}$. The second part is the $t$-derivative term in (3.7). Since we are considering the static case where all fluctuations are independent of $t$, it is zero. The last two parts are from the $x$ - and $y$-derivative terms in (3.7): that involving the sound mode and tensor mode fluctuations

$$
\begin{aligned}
\frac{1}{2 \kappa^{2}} \int d^{4} x\{- & \frac{1}{2}\left(\frac{\partial^{2}}{\partial x^{2}}+\frac{\partial^{2}}{\partial y^{2}}\right)\left[2 h_{t}^{t}(\vec{x}, z)+h_{x}^{x}(\vec{x}, z)+h_{y}^{y}(\vec{x}, z)\right] \\
& \left.+\frac{1}{2}\left(\frac{\partial^{2}}{\partial x^{2}}-\frac{\partial^{2}}{\partial y^{2}}\right)\left[h_{x}^{x}(\vec{x}, z)-h_{y}^{y}(\vec{x}, z)\right]+2 \frac{\partial^{2}}{\partial x \partial y} h_{y}^{x}(\vec{x}, z)\right\}
\end{aligned}
$$

is quadratic in derivatives, thus is of higher order. So the only relevant part is that involving the vector mode fluctuations

$$
\begin{aligned}
S^{(1)}=\frac{1}{2 \kappa^{2}} \int d^{4} x\{ & \frac{\lambda \Phi(z) A(z)}{F(z)}\left[-\left(\frac{\partial}{\partial x} a_{t}^{\mathbf{2}}(\vec{x}, z)\right)+\left(\frac{\partial}{\partial y} a_{t}^{\mathbf{1}}(\vec{x}, z)\right)\right] \\
+ & \left.\frac{\lambda A(z)^{2}}{r(z)^{2}}\left[-\left(\frac{\partial}{\partial x} a_{y}^{\mathbf{3}}(\vec{x}, z)\right)+\left(\frac{\partial}{\partial y} a_{x}^{\mathbf{3}}(\vec{x}, z)\right)\right]\right\} .
\end{aligned}
$$

Since this action is already linear in spatial derivatives (momentum), to solve the bulk fields $a_{t}^{1}, a_{t}^{2}, a_{x}^{3}$ and $a_{y}^{3}$ as a response to boundary source $\bar{h}_{t x}$ and $\bar{h}_{t y}$ up to the leading order, we only need to work in the zero momentum limit $\vec{k}=0$ and $\omega=0$, which significantly simplifies the EOMs.

\subsection{Vector mode EOMs}

We will work under the bulk gauge condition

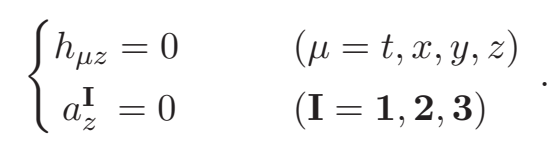

We first make some redefinitions of the coordinates and fields. In the rest of this section, unless otherwise stated, we will let $i, j=e, o$ exclusively. ${ }^{9}$ Define

$$
\begin{aligned}
\frac{1}{2}\left(h_{t x}+h_{t y}\right) & \equiv r(z)^{2} h_{t}^{e}, & \frac{1}{2}\left(h_{t x}-h_{t y}\right) & \equiv r(z)^{2} h_{t}^{o}, \\
\frac{1}{2}\left(a_{t}^{1}+a_{t}^{2}\right) & \equiv a_{t}^{e}, & \frac{1}{2}\left(a_{t}^{1}-a_{t}^{2}\right) & \equiv a_{t}^{o}, \\
\frac{1}{2}\left(a_{x}^{3}+a_{y}^{3}\right) & \equiv a_{e}^{3}, & \frac{1}{2}\left(a_{x}^{3}-a_{y}^{3}\right) & \equiv a_{o}^{\mathbf{3}} .
\end{aligned}
$$

\footnotetext{
${ }^{9}$ The indices $i, j=e, o$ are just short-hand notations introduced to make the equations look more compact. It is not necessary to think them as some co-variant indices that are raised and lowered by some metric. In other words, equations containing $i, j=e, o$ are just components of some covariant equations and themselves not covariant in some $(e, o)$-space. By definition, repeated indices of $i, j$ are summed over $e, o$.
} 
Fields in the left (right) column in the above definitions are even (odd) under the joint parity operations $x \leftrightarrow y$ and $\mathbf{1} \leftrightarrow \mathbf{2}$. In the limit $\omega=0$ and $\vec{k}=0$ these two groups decouple from each other. The independent equations are

$$
\begin{aligned}
\frac{d}{d z}\left[r(z)^{4}\left(\frac{d}{d z} h_{t}^{i}(z)\right)\right]+r(z)^{2}\left(\frac{d}{d z} \Phi(z)\right)\left(\frac{d}{d z} a_{i}^{\mathbf{3}}(z)\right) & =S_{t}^{(h) i}(z), \\
r(z)^{2}\left(\frac{d}{d z} \Phi(z)\right)\left(\frac{d}{d z} h_{t}^{i}(z)\right)+\frac{d}{d z}\left[F(z)\left(\frac{d}{d z} a_{i}^{\mathbf{3}}(z)\right)\right] & =S_{i}^{\mathbf{3}}(z), \\
\frac{d}{d z}\left(\frac{a_{t}^{i}(z)}{\Phi(z)}\right) & =S_{t}^{(a) i}(z),
\end{aligned}
$$

where

$$
\begin{aligned}
S_{t}^{(h) i}(z)= & {\left[r(z)^{2}\left(\frac{d}{d z} A(z)\right)^{2}+\frac{\lambda^{2}}{F(z)} A(z)^{4}\right] h_{t}^{i}(z)-\frac{\lambda^{2} \Phi(z)}{F(z)} A(z)^{2} a_{i}^{\mathbf{3}}(z) } \\
& -\left[r(z)^{2}\left(\frac{d}{d z} A(z)\right)\left(\frac{d}{d z} a_{t}^{i}(z)\right)+\frac{\lambda^{2}}{F(z)} A(z)^{3} a_{t}^{i}(z)\right], \\
S_{i}^{\mathbf{3}}(z)= & -\frac{\lambda^{2} \Phi(z)}{F(z)} A(z)^{2} h_{t}^{i}(z)+\frac{\lambda^{2}}{r(z)^{2}} A(z)^{2} a_{i}^{\mathbf{3}}(z)+\frac{\lambda^{2} \Phi(z)}{F(z)} A(z) a_{t}^{i}(z), \\
S_{t}^{(a) i}(z)= & {\left[\frac{d}{d z}\left(\frac{A(z)}{\Phi(z)}\right)\right] h_{t}^{i}(z)+\frac{F(z)}{r(z)^{2} \Phi(z)^{2}}\left[\left(\frac{d}{d z} A(z)\right) a_{i}^{\mathbf{3}}(z)-A(z)\left(\frac{d}{d z} a_{i}^{\mathbf{3}}(z)\right)\right], }
\end{aligned}
$$

and all other equations involving vector mode fields are linear combinations of the above equations and their $z$-derivatives. Notice that all sources $S_{\ldots} \ldots(z)$ in the above equations contain $A(z)$. So when we treat $A(z)$ perturbatively, all sources can also be treated perturbatively, and at the leading order they all vanish.

\subsection{Boundary-to-bulk propagators}

From (6.13) we can see that the two terms in the first line are proportional to $A(z)$ and those in the second line to $A(z)^{2}$, thus to obtain the leading order result for $S^{(1)}$, which is of order $A(z)^{2}$, we only need to solve $h_{t}^{i}$ and $a_{i}^{\mathbf{3}}$ to zeroth order in (independent of) $A(z)$ and $a_{t}^{i}$ to linear order in $A(z)$. First we solve the zeroth order equations for $h_{t}^{i}$ and $a_{i}^{\mathbf{3}}$ :

$$
\begin{aligned}
& \frac{d}{d z}\left[r(z)^{4}\left(\frac{d}{d z} h_{t}^{i}(z)\right)\right]+r(z)^{2}\left(\frac{d}{d z} \Phi(z)\right)\left(\frac{d}{d z} a_{i}^{\mathbf{3}}(z)\right)=0, \\
& r(z)^{2}\left(\frac{d}{d z} \Phi(z)\right)\left(\frac{d}{d z} h_{t}^{i}(z)\right)+\frac{d}{d z}\left[F(z)\left(\frac{d}{d z} a_{i}^{\mathbf{3}}(z)\right)\right]=0 .
\end{aligned}
$$

For each $i=e, o$ these are two coupled second order homogeneous ODEs, so they have four independent solutions. Two solutions are trivial to see: $h_{t}^{i}=$ constant, $a_{i}^{3}=0$ and $h_{t}^{i}=0$, $a_{i}^{\mathbf{3}}=$ constant. For the other two solutions, using (4.8) to solve these equations near the horizon, we find one of them contains $\ln \left(z-z_{H}\right)$ in $a_{i}^{\mathbf{3}}$ so it is dropped by the regularity requirement near the horizon. The last independent solution is

$$
\left\{\begin{array}{l}
h_{t}^{i}(z)=\frac{F(z)}{r(z)^{2}} \times \text { constant } \\
a_{i}^{\mathbf{3}}(z)=-\Phi(z) \times \text { same constant }
\end{array}\right.
$$


This can be checked by using the background equations (4.2)-(4.4). In appendix B we show that this solution is of a pure-gauge form which can be obtained by a residual gauge transformation. We require that $h_{t}^{i}(z)$ vanishes at the horizon. The same condition has been used in $[68,69]$. Bearing in mind that we only turn on $\bar{h}_{t i}$ source, ${ }^{10}$ we have at the leading order

$$
\left\{\begin{array}{l}
h_{t}^{i}(\vec{x}, z)=\left(\frac{F(z)}{r(z)^{2}}+O(\vec{\partial}, A(z))\right) \bar{h}_{t}^{i}(\vec{x}) \\
a_{i}^{\mathbf{3}}(\vec{x}, z)=\left(\Phi_{0}-\Phi(z)+O(\vec{\partial}, A(z))\right) \bar{h}_{t}^{i}(\vec{x})
\end{array} .\right.
$$

Next we solve for $a_{t}^{i}(z)$. At the leading order

$$
\frac{d}{d z}\left(\frac{a_{t}^{i}(z)}{\Phi(z)}\right)=0
$$

which has solution $a_{t}^{i}(z) \propto \Phi(z)$. This solution is also dropped because $\bar{a}_{t}^{i}$ source is not turned on. At the next order the source term becomes

$$
S_{t}^{(a) i}(z)=\frac{\Phi_{0} F(z)}{\Phi(z)^{2} r(z)^{2}}\left(\frac{d}{d z} A(z)\right) \bar{h}_{t}^{i}+O\left(A(z)^{2}\right),
$$

Requiring that $a_{t}^{i}(z) \rightarrow 0$ near the boundary, the solution is

$$
a_{t}^{i}(\vec{x}, z)=\left[\Phi_{0} \Phi(z) \int_{\infty}^{z} d \xi \frac{F(\xi)}{r(\xi)^{2} \Phi(\xi)^{2}}\left(\frac{d}{d \xi} A(\xi)\right)+O\left(\vec{\partial}, A(z)^{2}\right)\right] \bar{h}_{t}^{i}(\vec{x}) .
$$

\subsection{Angular momentum density and ratio to Hall viscosity}

From now on we restore the convention that $i, j=x, y$ used at the beginning of this section. Plug in the above solutions into (6.13), we have

$$
\begin{aligned}
S^{(1)}= & -\frac{\lambda}{2 \kappa^{2}} \int d^{3} x \int_{z_{H}}^{\infty} d z\left\{\left[\Phi_{0} \frac{\Phi(z)^{2} A(z)}{F(z)} \int_{\infty}^{z} d \xi \frac{F(\xi)}{r(\xi)^{2} \Phi(\xi)^{2}}\left(\frac{d}{d \xi} A(\xi)\right)\right.\right. \\
& \left.\left.+\left(\Phi_{0}-\Phi(z)\right) \frac{A(z)^{2}}{r(z)^{2}}\right] \epsilon^{i j} \partial_{i} \bar{h}_{t j}(\vec{x})+O\left(\vec{\partial}^{2}, A(z)^{3}\right)\right\} .
\end{aligned}
$$

Use (4.5) to integrate by parts the first term, and then compare with (6.9), we find the angular momentum density is

$$
\ell=-\frac{\lambda}{\kappa^{2}} \int_{z_{H}}^{\infty} d z\left[\frac{\Phi_{0} F(z)^{2}}{\lambda^{2} \Phi(z)^{2} r(z)^{2}}\left(\frac{d}{d z} A(z)\right)^{2}+\left(\Phi_{0}-\Phi(z)\right) \frac{A(z)^{2}}{r(z)^{2}}\right]+O\left(\vec{\partial}, A(z)^{3}\right)
$$

where $\Phi_{0}=\Phi(z=\infty)$.

\footnotetext{
${ }^{10}$ We view the boundary fields $\bar{h}_{t x}$ and $\bar{h}_{t}^{x}$ etc are living in the 3-dimensional flat Minkowskian space where the fluid (field theory) system lives. That means the indices of these boundary fields are raised and lowered by 3-dimensional flat Minkowskian metric, not by the 4-dimensional bulk metric (4.1).
} 
To compute the above expressions, again we set $A(z)=A^{(1)}(z), r(z)=r^{(0)}(z), F(z)=$ $F^{(0)}(z), \Phi(z)=\Phi^{(0)}(z)$ and use the analytic solutions obtained via variational method in the previous two sections. For canonical ensemble, we have

$$
\begin{aligned}
& \frac{\ell}{\rho}=-\frac{2.16}{\lambda R}\left[1-\frac{1.00}{(\lambda R)^{2}}+O\left(\frac{1}{(\lambda R)^{4}}\right)\right]\left(1-\frac{T}{T_{c}}\right) \theta\left(T_{c}-T\right), \\
& \frac{\ell}{s}=-\frac{1.27}{(\lambda R)^{2}}\left[1-\frac{1.18}{(\lambda R)^{2}}+O\left(\frac{1}{(\lambda R)^{4}}\right)\right]\left(1-\frac{T}{T_{c}}\right) \theta\left(T_{c}-T\right) .
\end{aligned}
$$

For Grand Canonical ensemble, we have

$$
\begin{aligned}
\frac{\ell}{\mu^{2}} & =-2.00\left[1-\frac{0.63}{(\lambda R)^{2}}+O\left(\frac{1}{(\lambda R)^{4}}\right)\right] \frac{R^{2}}{\kappa^{2}}\left(1-\frac{T}{T_{c}}\right) \theta\left(T_{c}-T\right), \\
\frac{\ell}{s} & =-\frac{1.08}{(\lambda R)^{2}}\left[1-\frac{0.99}{(\lambda R)^{2}}+O\left(\frac{1}{(\lambda R)^{4}}\right)\right]\left(1-\frac{T}{T_{c}}\right) \theta\left(T_{c}-T\right) .
\end{aligned}
$$

In (4.59) and (4.60) we have shown that the condensate $\langle\mathcal{O}\rangle \sim \sqrt{T_{c}-T}$, which implies the superfluid density $n_{s} \sim\langle\mathcal{O}\rangle^{2} \sim T_{c}-T$. This is the standard behavior one would expect from Ginzburg-Landau theory. Here we also have $\ell \sim T_{c}-T$, which gives

$$
\frac{\ell}{n_{s}} \sim \text { constant }
$$

This can be understood as a statement that each Cooper pair possesses a fixed amount of angular momentum. For a $p_{x}+i p_{y}$-wave Cooper pair, this number shall be just $\hbar=1$. For us, the precise value depends on the normalization constant in the relation $n_{s} \sim\langle\mathcal{O}\rangle^{2}$, which we will not determine explicitly here.

At the end, we find the ratio between Hall viscosity and angular momentum density to be

$$
\frac{\eta_{H}}{\ell}=-0.504\left[1+\frac{0.32}{(\lambda R)^{2}}+O\left(\frac{1}{(\lambda R)^{4}}\right)\right]
$$

from both ensembles. The minus sign is also in agreement with [7], but it can differ if the angular momentum or Hall viscosity is defined up to a sign. At large $\lambda R$, which corresponds to the probe limit regime where back-reactions to the metric can be neglected, the magnitude of this ratio is numerically $1 / 2$. As $\lambda R$ drops, the magnitude of the ratio increases. In the next section, we will see that at low temperature near the critical $\lambda_{c} R \approx 1$, it diverges logarithmically as $\ln T$ following the same behavior of Hall viscosity.

\section{Low temperature limit}

\subsection{About the complete phase diagram}

In this section, we investigate the low temperature limit of the holographic $p_{x}+i p_{y}$ model. This is also the small $\lambda$ limit along the critical regime, since $T_{c}$ is a monotonic increasing function of $\lambda$ as can be seen from the phase diagrams in figure 1. The corresponding gravity dual is the near-extremal limit of the AdS-RN black hole (4.27) and its hairy brother, and at the leading order, the extremal limit (4.30) represents $T \rightarrow 0$ limit. This is the low 
temperature limit of the holographic $p_{x}+i p_{y}$ phase, but may not be the dual of the low temperature limit of the actual field theory/condensed matter systems, due to various instabilities. So before we start the computation at the near-extremal limit, we would like to briefly comment on what we do not consider here.

The phase diagrams of figure 1 may not be the complete phase diagrams, because they are obtained by assuming that the AdS-RN black hole and its hairy version discussed in section 4 are the only two possible competing ground states of the system. At low temperature this is usually not the case, but even so, there are still complications. In section 4 , we have obtained that the characteristic function differences between the two phases are quadratic in $T_{c}-T$, and concluded that the phase transition along the critical line is second order. But there is an assumption made implicitly to reach such a conclusion, that is, the coefficient $a^{(2)}$ in (4.56) is always positive. However, as can be seen from (4.56), at some small $\lambda R \sim 2, a^{(2)}$ can become zero and then negative as $\lambda$ decreases. The consequence is that $\langle\mathcal{O}\rangle$ as a function of $T$ becomes multivalued near $T_{c}$, thus the phase transition becomes first order. ${ }^{11}$ This phenomena has been observed in holographic $s$-wave models $[33,100]$ and $p$-wave models $[101,102]$. Here we see this can happen in $p_{x}+i p_{y}$ model as well.

Another class of complications arises concerning the Hawking-Page transition between pure AdS and AdS-black hole backgrounds and their instabilities [103-108]: at low temperature, AdS-type backgrounds can be thermodynamically favored over black hole backgrounds. This is also important for zero temperature limit, because an extremal black hole has non-vanishing horizon area thus non-zero entropy, which implies the ground state is degenerate. But the real ground state shall be non-degenerate, thus it can not be described by an extremal black hole, but an AdS-type background with vanishing horizon area. In field theory language, the phase transition between AdS and black hole is a confinement/deconfinement transition, and in condensed matter language, insulator/conductor transition. The low/zero temperature limit of holographic $s$-wave models have been studied based on AdS domain wall geometries in [109-113] and on AdS solitons in [114-116], and that of the anisotropic $p$-wave model based on AdS domain walls in $[117,118]$ and on AdS solitons in $[119,120]$. So far we have not seen any study on the isotropic $p_{x}+i p_{y}$ model, possibly because of the instability discussed in [35].

In the following, we will ignore all these complications. If the actual low temperature state of the model can be a $p$-wave model which breaks isotropy, then the hydrodynamic analysis presented at the beginning of this paper, and the formula (2.14), will be invalid. The notion of Hall viscosity itself may not be even useful or well-defined if isotropy is lost. The main purpose of studying the near extremal limit of $p_{x}+i p_{y}$ model is to see how the results we have presented in the previous sections, mostly as series expansions for large $\lambda R$, can be extrapolated down to small $\lambda R$ regime to give an overall qualitative picture for all values of $\lambda R$. For this purpose, it is reasonable to ignore all the complications mentioned above.

\footnotetext{
${ }^{11}$ However, in the calculation for the near-extremal limit to be given later in this section, we find $a^{(2)}$ is still positive, so the phase transition is still second order there.
} 


\subsection{Near-extremal AdS-RN black hole with condensate}

For the low temperature limit, if we directly work with the extremal limit of AdS-RN black hole, we will encounter near-horizon divergence for the angular momentum density. To understand its origin and how to handle it properly, let us first have a look at the near-extremal limit. The near-extremal limit of (4.27) corresponds to take the following limit of the parameters:

$$
\left\{\begin{aligned}
q & =2 \sqrt{3-6 \tau} \lambda R \\
T & =\frac{3 z_{H}}{2 \pi R^{2}} \tau
\end{aligned} \quad \text { with } \quad \tau \ll 1 .\right.
$$

Then the inner horizon is

$$
z_{h}=z_{H}(1-\tau)
$$

The metric component $F^{(0)}(z)$ becomes

$$
F^{(0)}(z)=\frac{\left(z-z_{H}\right)\left(z-z_{h}\right)\left(z^{2}+2 z_{H} z+3 z_{H}^{2}\right)}{z^{2} R^{2}} .
$$

Now if we look at (6.29), near the horizon $z=z_{H}$ we encounter integrals like

$$
\int \frac{d z}{z-z_{h}}=\ln \left(z-z_{h}\right)
$$

When we evaluate the integral at the lower bound $z=z_{H}$, we get $\ln \left(z_{H}-z_{h}\right) \sim \ln (\tau)$. So if we work directly with the extremal black hole with $\tau=0$ from the very beginning, we will encounter divergence. ${ }^{12}$ So we see $\tau$ serves as a near-horizon regulator. If we choose to start with the extremal black hole, the solution will be that when we encounter such a divergence near the horizon, instead of setting the lower bound of the integral to be at $z=z_{H}$, we set it at $z=z_{H}(1+\tau)$. At the leading order this strategy will give us the same results as we work in near-extremal limit. This is what we will do in the following.

\subsection{Extremal AdS-RN black hole with condensate}

Now we can safely start with the extremal AdS-Reissner-Nordström Black Hole

$$
\left\{\begin{array}{l}
r^{(0)}(z)=\frac{z}{R} \\
F^{(0)}(z)=\frac{\left(z-z_{H}\right)^{2}\left(z^{2}+2 z_{H} z+3 z_{H}^{2}\right)}{z^{2} R^{2}} \\
\Phi^{(0)}(z)=\frac{2 \sqrt{3} z_{H}}{R}\left(1-\frac{z_{H}}{z}\right)
\end{array}\right.
$$

\footnotetext{
${ }^{12}$ Actually, $\ln \left(z-z_{h}\right)$ has already appear earlier. If we go through the same procedures as outlined in section 4 to solve the model analytically, we will get $\ln \left(z-z_{h}\right)$ when we solve $r^{(1)}(z), F^{(1)}(z)$ and $\Phi^{(1)}(z)$ using the integrals listed in appendix $\mathrm{C}$, as well as in $A^{(2)}(z)$. But except for $r^{(1)}(z), \ln \left(z-z_{h}\right)$ in the other functions is always multiplied by some factors of $z-z_{H}$ or $z-z_{h}$. So when evaluated at $z=z_{H}, \ln (\tau)$ will always drop off or appear in higher order, thus if we start directly with extremal black hole, we will not encounter divergence at these intermediate steps, until we reach $\ell$ in (6.29).
} 
then we will follow closely the same analytic procedure we have used in the previous sections. To avoid redundancy and repetition, we will only outline the differences here. The trial functions we use for $A^{(i)}(z)$ are:

$$
\begin{aligned}
& A^{(1)}(z)=\alpha_{1}^{(1)}\left(1+2 \frac{z_{H}}{z}+3 \frac{z_{H}^{2}}{z^{2}}\right)\left(c_{0}^{(1)}+\frac{1-2 z_{H} c_{0}^{(1)}}{z}+\sum_{i=2}^{n} \frac{c_{i}^{(1)}}{z^{2}}\right) \\
& A^{(2)}(z)=\left(1+2 \frac{z_{H}}{z}+3 \frac{z_{H}^{2}}{z^{2}}\right)\left(\alpha_{0}^{(2)}-\frac{2 z_{H} \alpha_{0}^{(2)}}{z}+\sum_{i=2}^{n} \frac{c_{i}^{(2)}}{z^{2}}\right)
\end{aligned}
$$

and $\tilde{A}^{(2)}(z)$ is just the version of the second line. From (4.35) we get the minimal coupling that can trigger a phase transition, i.e. the critical coupling, is

$$
\lambda_{c} R=0.745
$$

Since the temperature is already very low, we will not parametrize the deviation from the critical line as $T_{c}-T$, but instead as $\lambda-\lambda_{c}$.

For Canonical ensemble, we obtain:

$$
\begin{aligned}
\frac{\langle\mathcal{O}\rangle}{\rho} & =0.939\left(\frac{\lambda}{\lambda_{c}}-1\right)^{\frac{1}{2}} \theta\left(\lambda-\lambda_{c}\right), \\
c_{0}^{(1)}+\tilde{c}_{0}^{(2)} & =-\frac{2.91}{\sqrt{\rho} R \kappa}\left(\frac{\lambda}{\lambda_{c}}-1\right) \theta\left(\lambda-\lambda_{c}\right),
\end{aligned}
$$

then

$$
\begin{aligned}
\frac{\eta_{H}}{\rho} & =-2.14 \ln (\tau)\left(\frac{\lambda}{\lambda_{c}}-1\right) \theta\left(\lambda-\lambda_{c}\right), \\
\frac{\eta_{H}}{s} & =-1.18 \ln (\tau)\left(\frac{\lambda}{\lambda_{c}}-1\right) \theta\left(\lambda-\lambda_{c}\right),
\end{aligned}
$$

and

$$
\begin{aligned}
& \frac{\ell}{\rho}=-3.73\left(\frac{\lambda}{\lambda_{c}}-1\right) \theta\left(\lambda-\lambda_{c}\right), \\
& \frac{\ell}{s}=-2.06\left(\frac{\lambda}{\lambda_{c}}-1\right) \theta\left(\lambda-\lambda_{c}\right),
\end{aligned}
$$

with

$$
\tau=\frac{T}{\sqrt{\hat{\rho}}}
$$

and $\hat{\rho}=\frac{\kappa^{2}}{(2 \pi)^{3} R^{2}} \rho$.

For Grand Canonical ensemble, we obtain:

$$
\begin{aligned}
\frac{\langle\hat{\mathcal{O}}\rangle}{\mu^{2}} & =4.37 \times 10^{-3}\left(\frac{\lambda}{\lambda_{c}}-1\right)^{\frac{1}{2}} \theta\left(\lambda-\lambda_{c}\right), \\
c_{0}^{(1)}+\tilde{c}_{0}^{(2)} & =-\frac{2.71}{\mu R^{2}}\left(\frac{\lambda}{\lambda_{c}}-1\right) \theta\left(\lambda-\lambda_{c}\right),
\end{aligned}
$$


then

$$
\begin{aligned}
& \frac{\eta_{H}}{\mu^{2}}=-2.47 \ln (\tau)\left(\frac{\lambda}{\lambda_{c}}-1\right) \theta\left(\lambda-\lambda_{c}\right), \\
& \frac{\eta_{H}}{s}=-1.18 \ln (\tau)\left(\frac{\lambda}{\lambda_{c}}-1\right) \theta\left(\lambda-\lambda_{c}\right),
\end{aligned}
$$

and

$$
\begin{aligned}
\frac{\ell}{\mu^{2}} & =-4.30 \frac{R^{2}}{\kappa^{2}}\left(\frac{\lambda}{\lambda_{c}}-1\right) \theta\left(\lambda-\lambda_{c}\right), \\
\frac{\ell}{s} & =-2.06\left(\frac{\lambda}{\lambda_{c}}-1\right) \theta\left(\lambda-\lambda_{c}\right),
\end{aligned}
$$

with

$$
\tau=\frac{T}{\mu}
$$

and $\hat{\mathcal{O}}=\frac{\kappa^{2}}{(2 \pi)^{3} R^{2}} \mathcal{O}$.

For both ensembles, we have

$$
\begin{aligned}
\frac{\eta_{H}}{l} & =0.573 \ln (\tau), \\
\frac{\eta}{s} & =\frac{1}{4 \pi}\left\{1+11.7\left(\frac{\lambda}{\lambda_{c}}-1\right) \theta\left(\lambda-\lambda_{c}\right)\right\} .
\end{aligned}
$$

\section{Conclusions and comments}

In this paper we have studied the spontaneous parity breaking effect of the holographic $p_{x}+i p_{y}$ model of [34]. We have proposed an analytic approach to solve such holographic models by taking into full consideration of back-reactions. The method we have shown here for computing the angular momentum density of the model is general and can be used for other holographic models as well. We obtain analytic expressions for Hall viscosity and angular momentum density near the critical regime and find that the relation (1.1) between them holds in the probe limit regime where back-reations to metric can be ignored. The effect of angular momentum density is to accumulate momentum on the edge of the fluid system. Thus an edge current of momentum is generated, and its intensity is proportional to the angular momentum density.

At the end we would like to make several comments on the results and the holographic $p_{x}+i p_{y}$ model we use in this paper:

1. The near-critical behavior of Hall viscosity we have found in $p$-wave model $\eta_{H} \propto\left(T_{c}-T\right)$, which is different from that found in gravitational Chern-Simons model $[62,64]$ where $\eta_{H} \propto \sqrt{T_{c}-T}$. The reason is that in the former case the condensate $A(z)$ enters the EOMs and thus the final results quadratically, while in the latter the condensate - the axion scalar - enters linearly. 
2. We have only studied the near-critical regime of the model, because only this regime can be computed by analytic approaches. It is natural to ask how Hall viscosity and angular momentum density behave deep inside the superconducting phase. The complication is that a finite non-vanishing $A(z)$ spoils the integrability of EOMs for the tensor mode fluctuations (5.3) and (5.4), thus it is hard to find a closed form for Hall viscosity written in term of $A(z)$ and others. Going deep inside the symmetry-breaking phase requires numeric techniques, so we will leave this to future exploration.

3. As can be seen from (5.53) and (6.29), results for Hall viscosity and angular momentum density do not have the feature of membrane paradigm, with which such quantities can be written solely in terms of near-horizon fields, as found in [62]. Our results are written as integrals of the condensate over the whole region outside the black hole horizon, up to the boundary. This means the IR degrees of freedom interact non-trivially with UV degrees of freedom.

4. It is well known that the physics of Hall conductivity can be described at the low energy effective theory level by a gauge Chern-Simons term and is related to the topological nature of the states. This is also the spirit of many holographic constructions. So is true for Hall viscosity, which can be describe by a Wen-Zee term $[12,15]$ in the presence of external magnetic field or a Gravitational Chern-Simons term [62] for pure thermal cases. However, in the holographic $p_{x}+i p_{y}$ model, there is no Chern-Simons term in the action and we do not see the topological structure at this level. It will be interesting to see how the Chern-Simons term can be induced at the parity-breaking ground state and what the topological structure looks like (if there is any). This may possibly shed some light on the second comments above, and on understanding the relation of holographic $p_{x}+i p_{y}$ model to topological quantum states.

It is also interesting to see whether other holographic models with (either explicitly or spontaneously) broken parity respect (1.1), once the angular momentum density is correctly computed. The gravitational Chern-Simons model studied in $[62,64]$ has recently been shown in [121] to possess an angular momentum density. There, Hall viscosity, angular momentum density and their ratio all have complicated behaviors (numerically, even though the analytic expressions look simple) below and near the critical temperature and far away from the relation (1.1). A comprehensive understanding of the generation of Hall viscosity and angular momentum in generic holographic models, particularly for the gapped phases, still requires future works.

\section{Acknowledgments}

We thank Wan-Zhe Feng, Yan He, Kathryn Levin, Hong Liu, Dung Xuan Nguyen, Hirosi Ooguri, Matthew Roberts, Gordon Semenoff, Misha Stephanov, Bogdan Stoica and Paul Wiegmann for useful discussions and comments. This work is supported, in part, by DOE grant DE-FG02-90ER-40560, NSF DMS-1206648 and a Simons Investigator grant from the Simons Foundation. 


\section{A Green's function}

We start with the general form of a linear second order inhomogeneous ODE

$$
\frac{d}{d z}\left[P(z)\left(\frac{d}{d z} \phi(z)\right)\right]+Q(z) \phi(z)=S(z),
$$

where $z \in[a, b]$ and $P(z), Q(z)$ and $S(z)$ are known functions of $z$. We assume $\Theta_{m}(z)$ $(m=<,>)$ are two independent solutions to the homogeneous equation

$$
\frac{d}{d z}\left[P(z)\left(\frac{d}{d z} \Theta_{m}(z)\right)\right]+Q(z) \Theta_{m}(z)=0
$$

and satisfy appropriate boundary conditions at the two boundaries:

$$
\left.\Theta_{<}(z)\right|_{z \rightarrow a}=A(z-a)^{\alpha},\left.\quad \Theta_{>}(z)\right|_{z \rightarrow b}=B(z-b)^{\beta} .
$$

The Green's function $G\left(z, z^{\prime}\right)$ satisfies similar boundary conditions and

$$
\frac{d}{d z}\left[P(z)\left(\frac{d}{d z} G\left(z, z^{\prime}\right)\right)\right]+Q(z) G\left(z, z^{\prime}\right)=\delta\left(z-z^{\prime}\right),
$$

where $\delta\left(z-z^{\prime}\right)$ is the Dirac delta function. Then we have

$$
\phi(z)=\int_{a}^{b} d z^{\prime} G\left(z, z^{\prime}\right) S\left(z^{\prime}\right) .
$$

The Green's function can be written in terms of the above two independent solutions:

$$
G\left(z, z^{\prime}\right)=\frac{1}{N_{r}}\left\{\Theta_{<}(z) \Theta_{>}\left(z^{\prime}\right) \theta\left(z^{\prime}-z\right)+\Theta_{<}\left(z^{\prime}\right) \Theta_{>}(z) \theta\left(z-z^{\prime}\right)\right\},
$$

and the normalization constant $N_{r}$ can be calculated by integrating (A.4) from $z=z_{-}^{\prime}$ to $z=z_{+}^{\prime}$, which gives

$$
N_{r}=P(z) \mathrm{Wr}(z)
$$

where the Wronskian is

$$
\operatorname{Wr}(z) \equiv \Theta_{<}(z)\left(\frac{d}{d z} \Theta_{>}(z)\right)-\Theta_{>}(z)\left(\frac{d}{d z} \Theta_{<}(z)\right) .
$$

One can easily show from (A.2) that $P(z) \mathrm{Wr}(z)$, even though it's a product of two functions of $z$, is indeed a constant independent of $z$, thus $N_{r}=$ constant. Assume near the boundary $z=b$ :

$$
\left\{\begin{aligned}
P(z) & \rightarrow P_{0}(z-b)^{\gamma} \\
\Theta_{<}(z) & \rightarrow B_{<}(z-b)^{\beta<} \\
\Theta_{>}(z) & \rightarrow B_{>}(z-b)^{\beta_{>}}
\end{aligned}\right.
$$

and $Q(z)$ are sub-leading to $P(z)$. The indicial equation of (A.2) gives

$$
\begin{aligned}
\beta_{<}, \beta_{>} & =0 \quad \text { or } \quad 1-\gamma, \\
\beta_{<}+\beta_{>} & =1-\gamma
\end{aligned}
$$


thus

$$
N_{r}=\left(\beta_{>}-\beta_{<}\right) P_{0} B_{<} B_{>} .
$$

The solution to the inhomogeneous equation is

$$
\phi(z)=\frac{1}{N_{r}}\left\{\Theta_{<}(z) \int_{z}^{b} d z^{\prime} \Theta_{>}\left(z^{\prime}\right) S\left(z^{\prime}\right)+\Theta_{>}(z) \int_{a}^{z} d z^{\prime} \Theta_{<}\left(z^{\prime}\right) S\left(z^{\prime}\right)\right\} .
$$

This is the formula we will use in the text to solve (5.4). Furthermore, one can integrate by parts the above expression to get an alternative version

$$
\begin{aligned}
\phi(z)= & \frac{1}{N_{r}}\left\{\Theta_{<}(z) \int_{b}^{z} d z^{\prime}\left(\frac{d}{d z^{\prime}} \Theta_{>}\left(z^{\prime}\right)\right) \int_{a}^{z^{\prime}} d z^{\prime \prime} S\left(z^{\prime \prime}\right)\right. \\
& \left.-\Theta_{>}(z) \int_{a}^{z} d z^{\prime}\left(\frac{d}{d z^{\prime}} \Theta_{<}\left(z^{\prime}\right)\right) \int_{a}^{z^{\prime}} d z^{\prime \prime} S\left(z^{\prime \prime}\right)+\Theta_{>}(b) \Theta_{<}(z) \int_{a}^{b} d z^{\prime} S\left(z^{\prime}\right)\right\} .
\end{aligned}
$$

We can see that when one $\Theta_{m}(z)$ is constant (when $Q(z)=0$, such as in (5.3)) this expression gives immediately the result as one obtains by directly integrating the equation.

\section{B Residual gauge transformations}

The gauge condition (6.14) does not completely fix the gauge. There are still residual gauge freedoms in the other non-vanishing components of $h_{\mu \nu}$ and $a_{\mu}^{\mathbf{I}}$. These remaining gauge freedoms are not strong enough to set any of these fields to zero, but they can be used to gauge away certain parts of them - the pure gauge solutions. The gauge transformations for Einstein-SU(2) system are

$$
\begin{aligned}
\delta g_{\mu \nu} & =-\nabla_{\mu} \xi_{\nu}-\nabla_{\nu} \xi_{\mu}, \\
\delta A_{\mu}^{\mathbf{I}} & =-\xi^{\nu} \nabla_{\nu} A_{\mu}^{\mathbf{I}}+A^{\mathbf{I} \nu} \nabla_{\mu} \xi_{\nu}-\nabla_{\mu} \Lambda^{\mathbf{I}}+\epsilon^{\mathbf{I J K}} A_{\mu}^{\mathbf{J}} \Lambda^{\mathbf{K}},
\end{aligned}
$$

where $\xi^{\mu}$ and $\Lambda^{\mathbf{I}}$ are the gauge parameters. Here we only consider the static case, where $\xi^{\mu}=\xi^{\mu}(x, y, z)$ and $\Lambda^{\mathbf{I}}=\Lambda^{\mathbf{I}}(x, y, z)$. The residual gauge transformations need to preserve the gauge condition (6.14), which means

$$
\begin{aligned}
0 & =\delta g_{t z}=F(z)\left(\frac{\partial}{\partial z} \xi^{t}\right) \\
0 & =\delta g_{x z}=-r(z)^{2}\left(\frac{\partial}{\partial z} \xi^{x}\right)-\frac{1}{F(z)}\left(\frac{\partial}{\partial x} \xi^{z}\right) \\
0 & =\delta g_{y z}=-r(z)^{2}\left(\frac{\partial}{\partial z} \xi^{y}\right)-\frac{1}{F(z)}\left(\frac{\partial}{\partial y} \xi^{z}\right) \\
0 & =\delta g_{z z}=\frac{1}{F(z)^{2}}\left[\left(\frac{d}{\partial z} F(z)\right) \xi^{z}-2 F(z)\left(\frac{\partial}{\partial z} \xi^{z}\right)\right] \\
0 & =\delta A_{z}^{\mathbf{1}}=-A(z)\left(\frac{\partial}{\partial z} \xi^{x}\right)-\left(\frac{\partial}{\partial z} \Lambda^{\mathbf{1}}\right) \\
0 & =\delta A_{z}^{2}=-A(z)\left(\frac{\partial}{\partial z} \xi^{y}\right)-\left(\frac{\partial}{\partial z} \Lambda^{2}\right) \\
0 & =\delta A_{z}^{3}=-\Phi(z)\left(\frac{\partial}{\partial z} \xi^{t}\right)-\left(\frac{\partial}{\partial z} \Lambda^{\mathbf{3}}\right)
\end{aligned}
$$


The solutions are

$$
\begin{aligned}
\xi^{t} & =\tilde{\xi}^{t}(x, y) \\
\xi^{x} & =-\int d z \frac{1}{r(z)^{2} \sqrt{F(z)}}\left(\frac{\partial}{\partial x} \tilde{\xi}^{z}(x, y)\right)+\tilde{\xi}^{x}(x, y), \\
\xi^{y} & =-\int d z \frac{1}{r(z)^{2} \sqrt{F(z)}}\left(\frac{\partial}{\partial y} \tilde{\xi}^{z}(x, y)\right)+\tilde{\xi}^{y}(x, y), \\
\xi^{z} & =\sqrt{F(z)} \tilde{\xi}^{z}(x, y) \\
\Lambda^{\mathbf{1}} & =\int d z \frac{A(z)}{r(z)^{2} \sqrt{F(z)}}\left(\frac{\partial}{\partial x} \tilde{\xi}^{z}(x, y)\right)+\tilde{\Lambda}^{\mathbf{1}}(x, y), \\
\Lambda^{\mathbf{2}} & =\int d z \frac{A(z)}{r(z)^{2} \sqrt{F(z)}}\left(\frac{\partial}{\partial y} \tilde{\xi}^{z}(x, y)\right)+\tilde{\Lambda}^{2}(x, y), \\
\Lambda^{\mathbf{3}} & =\tilde{\Lambda}^{\mathbf{3}}(x, y),
\end{aligned}
$$

where $\tilde{\xi}^{\mu}(x, y)$ is an arbitrary vector function of $(x, y)$. Then the residual gauge transformations for the vector mode fluctuations are

$$
\begin{aligned}
\delta g_{t x}= & F(z)\left(\frac{\partial}{\partial x} \tilde{\xi}^{t}(x, y)\right) \\
\delta A_{x}^{\mathbf{3}}= & -\Phi(z)\left(\frac{\partial}{\partial x} \tilde{\xi}^{t}(x, y)\right)+\lambda A(z) \int d z \frac{A(z)}{r(z)^{2} \sqrt{F(z)}}\left(\frac{\partial}{\partial y} \tilde{\xi}^{z}(x, y)\right) \\
& +\lambda A(z) \tilde{\Lambda}^{2}(x, y)-\left(\frac{\partial}{\partial x} \tilde{\Lambda}^{3}(x, y)\right), \\
\delta A_{t}^{\mathbf{1}}= & -\lambda \Phi(z)\left[\int d z \frac{A(z)}{r(z)^{2} \sqrt{F(z)}}\left(\frac{\partial}{\partial y} \tilde{\xi}^{z}(x, y)\right)+\tilde{\Lambda}^{\mathbf{2}}(x, y)\right],
\end{aligned}
$$

and

$$
\begin{aligned}
\delta g_{t y}= & F(z)\left(\frac{\partial}{\partial y} \tilde{\xi}^{t}(x, y)\right) \\
\delta A_{y}^{\mathbf{3}}= & -\Phi(z)\left(\frac{\partial}{\partial y} \tilde{\xi}^{t}(x, y)\right)-\lambda A(z) \int d z \frac{A(z)}{r(z)^{2} \sqrt{F(z)}}\left(\frac{\partial}{\partial x} \tilde{\xi}^{z}(x, y)\right) \\
& -\lambda A(z) \tilde{\Lambda}^{\mathbf{1}}(x, y)-\left(\frac{\partial}{\partial y} \tilde{\Lambda}^{3}(x, y)\right) \\
\delta A_{t}^{\mathbf{1}=} & \lambda \Phi(z)\left[\int d z \frac{A(z)}{r(z)^{2} \sqrt{F(z)}}\left(\frac{\partial}{\partial x} \tilde{\xi}^{z}(x, y)\right)+\tilde{\Lambda}^{\mathbf{1}}(x, y)\right] .
\end{aligned}
$$

In the above equations, the part that contains $\tilde{\xi}^{t}(x, y)$ is the residual gauge transformation that can be used to obtain the fourth solution (6.24) in the vector mode boundary-to-bulk propagators.

\section{Near-critical first order fields}

Here we give the solutions for $r^{(1)}(z), F^{(1)}(z)$ and $\Phi^{(1)}(z)$. They are written as indefinite integrals over sources quadratic in $A^{(1)}(z)$, plus two general solutions to the homogeneous 
equations with arbitrary coefficients $C_{1}-C_{6}$, as to be used in section 4.7.

$$
\begin{aligned}
r^{(1)}(z)= & -\frac{1}{2} \int d z \int d z\left[\frac{1}{r^{(0)}(z)}\left(\frac{d}{d z} A^{(1)}(z)\right)^{2}+\frac{\lambda^{2} \Phi^{(0)}(z)^{2}}{r^{(0)}(z) F^{(0)}(z)^{2}} A^{(1)}(z)^{2}\right] \\
& +C_{1} z+C_{2}, \\
\Phi^{(1)}(z)= & -2 \int \frac{d z}{r^{(0)}(z)^{2}} \int d z\left\{\left(\frac{d}{d z} \Phi^{(0)}(z)\right)\left[r^{(0)}(z)\left(\frac{d}{d z} r^{(1)}(z)\right)-\left(\frac{d}{d z} r^{(0)}(z)\right) r^{(1)}(z)\right]\right. \\
& \left.-\frac{\lambda^{2} \Phi^{(0)}(z)}{F^{(0)}(z)} A^{(1)}(z)^{2}\right\}+C_{3} \int \frac{d z}{r^{(0)}(z)^{2}}+C_{4}, \\
F^{(1)}(z)= & \int \frac{d z}{r^{(0)}(z)^{2}} \int d z\left\{-2 r^{(0)}(z) \frac{d}{d z}\left[F^{(0)}(z)\left(\frac{d}{d z} r^{(1)}(z)\right)\right]\right. \\
& \left.+2\left(\frac{d}{d z} r^{(0)}(z)\right)\left(\frac{d}{d z} F^{(0)}(z)\right) r^{(1)}(z)+r^{(0)}(z)^{2}\left(\frac{d}{d z} \Phi^{(0)}(z)\right)\left(\frac{d}{d z} \Phi^{(1)}(z)\right)\right\} \\
& +C_{5} \int \frac{d z}{r^{(0)}(z)^{2}}+C_{6} .
\end{aligned}
$$

The trace equation (4.6) at $O\left(\epsilon^{2}\right)$ order is

$$
\begin{aligned}
& \frac{1}{r^{(0)}(z)} \frac{d}{d z}\left[r^{(0)}(z)^{4}\left(\frac{d}{d z} F^{(1)}(z)\right)\right]+4 r^{(0)}(z) \frac{d}{d z}\left[r^{(0)}(z) F^{(0)}(z)\left(\frac{d}{d z} r^{(1)}(z)\right)\right] \\
& +2 r^{(0)}(z)\left(\frac{d}{d z} r^{(0)}(z)\right)^{2} F^{(1)}(z)-4\left(\frac{d}{d z} r^{(0)}(z)\right)\left[\frac{d}{d z}\left(r^{(0)}(z) F^{(0)}(z)\right)\right] r^{(1)}(z)=0 .
\end{aligned}
$$

Open Access. This article is distributed under the terms of the Creative Commons Attribution License (CC-BY 4.0), which permits any use, distribution and reproduction in any medium, provided the original author(s) and source are credited.

\section{References}

[1] T.L. Hughes, R.G. Leigh and O. Parrikar, Torsional Anomalies, Hall Viscosity and Bulk-boundary Correspondence in Topological States, Phys. Rev. D 88 (2013) 025040 [arXiv: 1211.6442] [INSPIRE].

[2] E.M. Lifshitz and L. P. Pitaevskii, Course of Theoretical Physics. Vol. 10: Physical Kinetics, Pergamon, Oxford U.K. (1981).

[3] J.E. Avron, R. Seiler and P.G. Zograf, Viscosity of quantum Hall fluids, Phys. Rev. Lett. 75 (1995) 697 [INSPIRE].

[4] J.E. Avron, Odd Viscosity, J. Stat. Phys. 92 (1998) 543 [physics/9712050].

[5] I.V. Tokatly and G. Vignale, Lorentz shear modulus of a two-dimensional electron gas at high magnetic field, Phys. Rev. B 76 (2007) 161305 [arXiv:0706.2454].

[6] I.V. Tokatly and G. Vignale, Lorentz shear modulus of fractional quantum Hall states, arXiv:0812.4331.

[7] N. Read, Non-Abelian adiabatic statistics and Hall viscosity in quantum Hall states and $p(x)+i p(y)$ paired superfluids, Phys. Rev. B 79 (2009) 045308 [arXiv:0805.2507] [INSPIRE]. 
[8] F.D.M. Haldane, 'Hall viscosity' and intrinsic metric of incompressible fractional Hall fluids, arXiv:0906.1854 [INSPIRE].

[9] N. Read and E.H. Rezayi, Hall viscosity, orbital spin, and geometry: paired superfluids and quantum Hall systems, Phys. Rev. B 84 (2011) 085316 [arXiv: 1008. 0210].

[10] T.L. Hughes, R.G. Leigh and E. Fradkin, Torsional Response and Dissipationless Viscosity in Topological Insulators, Phys. Rev. Lett. 107 (2011) 075502 [arXiv:1101.3541] [INSPIRE].

[11] B. Bradlyn, M. Goldstein and N. Read, Kubo formulas for viscosity: Hall viscosity, Ward identities and the relation with conductivity, Phys. Rev. B 86 (2012) 245309 [arXiv:1207.7021] [INSPIRE].

[12] C. Hoyos and D.T. Son, Hall Viscosity and Electromagnetic Response, Phys. Rev. Lett. 108 (2012) 066805 [arXiv:1109.2651] [InSPIRE].

[13] A. Nicolis and D.T. Son, Hall viscosity from effective field theory, arXiv:1103.2137 [INSPIRE].

[14] C. Hoyos, S. Moroz and D.T. Son, Effective theory of chiral two-dimensional superfluids, Phys. Rev. B 89 (2014) 174507 [arXiv: 1305.3925] [INSPIRE].

[15] D.T. Son, Newton-Cartan Geometry and the Quantum Hall Effect, arXiv:1306.0638 [INSPIRE].

[16] Y. Hidaka, Y. Hirono, T. Kimura and Y. Minami, Viscoelastic-electromagnetism and Hall viscosity, PTEP 2013 (2013) 013A02 [arXiv:1206. 0734] [INSPIRE].

[17] P.B. Wiegmann, Quantum Hydrodynamics of Fractional Hall Effect: Quantum Kirchhoff Equations, arXiv:1211.5132.

[18] P.B. Wiegmann, Anomalous Hydrodynamics of Fractional Quantum Hall States, JETP 144 (2013) 617 [arXiv: 1305.6893].

[19] P.B. Wiegmann, Hydrodynamics of Euler incompressible fluid and the Fractional Quantum Hall Effect, arXiv:1309.5992.

[20] X.G. Wen and A. Zee, Shift and spin vector: New topological quantum numbers for the Hall fluids, Phys. Rev. Lett. 69 (1992) 953 [Erratum ibid. 69 (1992) 3000] [INSPIRE].

[21] J.M. Maldacena, The large- $N$ limit of superconformal field theories and supergravity, Adv. Theor. Math. Phys. 2 (1998) 231 [Int. J. Theor. Phys. 38 (1999) 1113] [hep-th/9711200] [INSPIRE].

[22] S.S. Gubser, I.R. Klebanov and A.M. Polyakov, Gauge theory correlators from noncritical string theory, Phys. Lett. B 428 (1998) 105 [hep-th/9802109] [INSPIRE].

[23] E. Witten, Anti-de Sitter space and holography, Adv. Theor. Math. Phys. 2 (1998) 253 [hep-th/9802150] [INSPIRE].

[24] G. Policastro, D.T. Son and A.O. Starinets, From AdS/CFT correspondence to hydrodynamics, JHEP 09 (2002) 043 [hep-th/0205052] [INSPIRE].

[25] G. Policastro, D.T. Son and A.O. Starinets, From AdS/CFT correspondence to hydrodynamics. 2. Sound waves, JHEP 12 (2002) 054 [hep-th/0210220] [INSPIRE].

[26] P. Kovtun, D.T. Son and A.O. Starinets, Holography and hydrodynamics: Diffusion on stretched horizons, JHEP 10 (2003) 064 [hep-th/0309213] [INSPIRE].

[27] S. Cremonini, The Shear Viscosity to Entropy Ratio: A Status Report, Mod. Phys. Lett. B 25 (2011) 1867 [arXiv:1108.0677] [INSPIRE]. 
[28] S.S. Gubser, Phase transitions near black hole horizons, Class. Quant. Grav. 22 (2005) 5121 [hep-th/0505189] [INSPIRE].

[29] S.S. Gubser, Breaking an Abelian gauge symmetry near a black hole horizon, Phys. Rev. D 78 (2008) 065034 [arXiv:0801.2977] [INSPIRE].

[30] S.A. Hartnoll, C.P. Herzog and G.T. Horowitz, Building a Holographic Superconductor, Phys. Rev. Lett. 101 (2008) 031601 [arXiv:0803.3295] [INSPIRE].

[31] S.A. Hartnoll, C.P. Herzog and G.T. Horowitz, Holographic Superconductors, JHEP 12 (2008) 015 [arXiv:0810.1563] [inSPIRE].

[32] G.T. Horowitz and M.M. Roberts, Holographic Superconductors with Various Condensates, Phys. Rev. D 78 (2008) 126008 [arXiv:0810.1077] [INSPIRE].

[33] C.P. Herzog, P.K. Kovtun and D.T. Son, Holographic model of superfluidity, Phys. Rev. D 79 (2009) 066002 [arXiv:0809.4870] [InSPIRE].

[34] S.S. Gubser, Colorful horizons with charge in anti-de Sitter space, Phys. Rev. Lett. 101 (2008) 191601 [arXiv:0803.3483] [InSPIRE].

[35] S.S. Gubser and S.S. Pufu, The Gravity dual of a p-wave superconductor, JHEP 11 (2008) 033 [arXiv:0805.2960] [INSPIRE].

[36] M.M. Roberts and S.A. Hartnoll, Pseudogap and time reversal breaking in a holographic superconductor, JHEP 08 (2008) 035 [arXiv: 0805.3898] [INSPIRE].

[37] J.-W. Chen, Y.-J. Kao, D. Maity, W.-Y. Wen and C.-P. Yeh, Towards A Holographic Model of D-Wave Superconductors, Phys. Rev. D 81 (2010) 106008 [arXiv:1003.2991] [InSPIRE].

[38] F. Benini, C.P. Herzog, R. Rahman and A. Yarom, Gauge gravity duality for d-wave superconductors: prospects and challenges, JHEP 11 (2010) 137 [arXiv:1007.1981] [INSPIRE].

[39] C.P. Herzog, Lectures on Holographic Superfluidity and Superconductivity, J. Phys. A 42 (2009) 343001 [arXiv:0904.1975] [inSPIRE].

[40] G.T. Horowitz, Introduction to Holographic Superconductors, Lect. Notes Phys. 828 (2011) 313 [arXiv:1002.1722] [INSPIRE].

[41] R.E. Prange and S.M. Girvin eds., The Quantum Hall Effect, second edition, Springer-Verlag, Heidelberg Germany (1990).

[42] S. Das Sarma and A. Pinczuk eds., Perspectives in Quantum Hall Effects, John Wiley and Sons, New York U.S.A. (1997).

[43] S.M. Girvin, The Quantum Hall Effect: Novel Excitations and Broken Symmetries, cond-mat/9907002.

[44] G. Murthy and R. Shankar, Hamiltonian theories of the fractional quantum Hall effect, Rev. Mod. Phys. 75 (2003) 1101 [InSPIRE].

[45] A. Karlhede, S.A. Kivelson and S.L. Sondhi, The Quantum Hall effect, the article in Correlated Electron Systems (Jerusalem Winter School in Theoretical Physics. Vol. 9), V.J. Emery eds., World Scientific, Singapore (1992).

[46] S.A. Hartnoll and P. Kovtun, Hall conductivity from dyonic black holes, Phys. Rev. D 76 (2007) 066001 [arXiv:0704.1160] [INSPIRE].

[47] E. Keski-Vakkuri and P. Kraus, Quantum Hall Effect in AdS/CFT, JHEP 09 (2008) 130 [arXiv:0805.4643] [INSPIRE]. 
[48] J.L. Davis, P. Kraus and A. Shah, Gravity Dual of a Quantum Hall Plateau Transition, JHEP 11 (2008) 020 [arXiv:0809.1876] [InSPIRE].

[49] M. Fujita, W. Li, S. Ryu and T. Takayanagi, Fractional Quantum Hall Effect via Holography: Chern-Simons, Edge States and Hierarchy, JHEP 06 (2009) 066 [arXiv: 0901.0924] [INSPIRE].

[50] O. Bergman, N. Jokela, G. Lifschytz and M. Lippert, Quantum Hall Effect in a Holographic Model, JHEP 10 (2010) 063 [arXiv: 1003.4965] [INSPIRE].

[51] E. Gubankova et al., Holographic fermions in external magnetic fields, Phys. Rev. D 84 (2011) 106003 [arXiv:1011.4051] [INSPIRE].

[52] C. Kristjansen and G.W. Semenoff, Giant D5 Brane Holographic Hall State, JHEP 06 (2013) 048 [arXiv: 1212.5609] [INSPIRE].

[53] J. Erdmenger, M. Haack, M. Kaminski and A. Yarom, Fluid dynamics of R-charged black holes, JHEP 01 (2009) 055 [arXiv: 0809.2488] [INSPIRE].

[54] N. Banerjee et al., Hydrodynamics from charged black branes, JHEP 01 (2011) 094 [arXiv: 0809.2596] [INSPIRE].

[55] A. Gynther, K. Landsteiner, F. Pena-Benitez and A. Rebhan, Holographic Anomalous Conductivities and the Chiral Magnetic Effect, JHEP 02 (2011) 110 [arXiv:1005.2587] [INSPIRE].

[56] T. Kalaydzhyan and I. Kirsch, Fluid/gravity model for the chiral magnetic effect, Phys. Rev. Lett. 106 (2011) 211601 [arXiv:1102.4334] [INSPIRE].

[57] I. Amado, K. Landsteiner and F. Pena-Benitez, Anomalous transport coefficients from Kubo formulas in Holography, JHEP 05 (2011) 081 [arXiv:1102.4577] [INSPIRE].

[58] K. Landsteiner, E. Megias and F. Pena-Benitez, Gravitational Anomaly and Transport, Phys. Rev. Lett. 107 (2011) 021601 [arXiv:1103.5006] [InSPIRE].

[59] K. Landsteiner, E. Megias, L. Melgar and F. Pena-Benitez, Holographic Gravitational Anomaly and Chiral Vortical Effect, JHEP 09 (2011) 121 [arXiv:1107.0368] [INSPIRE].

[60] K. Landsteiner and L. Melgar, Holographic Flow of Anomalous Transport Coefficients, JHEP 10 (2012) 131 [arXiv:1206.4440] [INSPIRE].

[61] K. Landsteiner, E. Megias and F. Pena-Benitez, Anomalous Transport from Kubo Formulae, Lect. Notes Phys. 871 (2013) 433 [arXiv:1207.5808] [INSPIRE].

[62] O. Saremi and D.T. Son, Hall viscosity from gauge/gravity duality, JHEP 04 (2012) 091 [arXiv:1103.4851] [INSPIRE].

[63] S. Alexander and N. Yunes, Chern-Simons Modified General Relativity, Phys. Rept. 480 (2009) 1 [arXiv:0907.2562] [INSPIRE].

[64] J.-W. Chen, N.-E. Lee, D. Maity and W.-Y. Wen, A Holographic Model For Hall Viscosity, Phys. Lett. B 713 (2012) 47 [arXiv:1110.0793] [INSPIRE].

[65] J.-W. Chen, S.-H. Dai, N.-E. Lee and D. Maity, Novel Parity Violating Transport Coefficients in 2+1 Dimensions from Holography, JHEP 09 (2012) 096 [arXiv:1206.0850] [INSPIRE].

[66] R.-G. Cai, T.-J. Li, Y.-H. Qi and Y.-L. Zhang, Incompressible Navier-Stokes Equations from Einstein Gravity with Chern-Simons Term, Phys. Rev. D 86 (2012) 086008 [arXiv: 1208.0658] [INSPIRE]. 
[67] D.-C. Zou and B. Wang, Holographic parity violating charged fluid dual to Chern-Simons modified gravity, arXiv:1306.5486 [INSPIRE].

[68] H. Liu, H. Ooguri, B. Stoica and N. Yunes, Spontaneous Generation of Angular Momentum in Holographic Theories, Phys. Rev. Lett. 110 (2013) 211601 [arXiv:1212.3666] [INSPIRE].

[69] H. Liu, H. Ooguri and B. Stoica, Angular Momentum Generation by Parity Violation, Phys. Rev. D 89 (2014) 106007 [arXiv:1311.5879] [InSPIRE].

[70] K. Jensen et al., Parity-Violating Hydrodynamics in 2+1 Dimensions, JHEP 05 (2012) 102 [arXiv:1112.4498] [INSPIRE].

[71] J. Sonner, A Rotating Holographic Superconductor, Phys. Rev. D 80 (2009) 084031 [arXiv: 0903.0627] [INSPIRE].

[72] M. Stone and R. Roy, Edge modes, edge currents, and gauge invariance in superfluids and superconductors, Phys. Rev. B 69 (2004) 184511 [cond-mat/0308034].

[73] J.A. Sauls, Surface States, Edge Currents and the Angular Momentum of Chiral-wave Superfluids, Phys. Rev. B 84 (2011) 214509 [arXiv:1209.5501].

[74] Y. Tsutsumi and K. Machida, Edge mass current and the role of Majorana fermions in a-phase superfluid He-3, Phys. Rev. B 85 (2012) 100506 [InSPIRE].

[75] T.M. Rice and M. Sigrist, $\mathrm{Sr}_{2} \mathrm{RuO}_{4}$ : an electronic analogue of ${ }^{3} \mathrm{He}$ ?, J. Phys. Cond. Matt. 7 (1995) 643.

[76] G. Baskaran, Why is $\mathrm{Sr}_{2} \mathrm{RuO}_{4}$ not a high $T_{c}$ superconductor? Electron correlation, Hund's coupling and p-wave instability, Physica B 223-224 (1996) 490.

[77] A. Stern, Anyons and the quantum Hall effect - A pedagogical review, Annals Phys. 323 (2008) 204 [arXiv:0711.4697].

[78] C. Nayak, S.H. Simon, A. Stern, M. Freedman and S. Das Sarma, Non-Abelian anyons and topological quantum computation, Rev. Mod. Phys. 80 (2008) 1083 [INSPIRE].

[79] R.L. Willett, The quantum Hall effect at $5 / 2$ filling factor, Rep. Prog. Phys. 76 (2013) 076501.

[80] E. Radu and D.H. Tchrakian, Stable black hole solutions with non-Abelian fields, Phys. Rev. D 85 (2012) 084022 [arXiv:1111.0418] [INSPIRE].

[81] M. Kaminski and S. Moroz, Non-Relativistic Parity-Violating Hydrodynamics in Two Spatial Dimensions, Phys. Rev. B 89 (2014) 115418 [arXiv:1310.8305] [InSPIRE].

[82] J. Bhattacharya, S. Bhattacharyya and S. Minwalla, Dissipative Superfluid dynamics from gravity, JHEP 04 (2011) 125 [arXiv:1101.3332] [INSPIRE].

[83] J. Bhattacharya, S. Bhattacharyya, S. Minwalla and A. Yarom, A Theory of first order dissipative superfluid dynamics, JHEP 05 (2014) 147 [arXiv:1105.3733] [INSPIRE].

[84] Y. Neiman and Y. Oz, Anomalies in Superfluids and a Chiral Electric Effect, JHEP 09 (2011) 011 [arXiv:1106.3576] [INSPIRE].

[85] S. Chapman, C. Hoyos and Y. Oz, Lifshitz Superfluid Hydrodynamics, JHEP 07 (2014) 027 [arXiv: 1402.2981] [INSPIRE].

[86] C. Hoyos, B.S. Kim and Y. Oz, Odd Parity Transport In Non-Abelian Superfluids From Symmetry Locking, arXiv:1404.7507 [INSPIRE].

[87] C.P. Herzog and D.T. Son, Schwinger-Keldysh propagators from AdS/CFT correspondence, JHEP 03 (2003) 046 [hep-th/0212072] [INSPIRE]. 
[88] E. Barnes, D. Vaman, C. Wu and P. Arnold, Real-time finite-temperature correlators from AdS/CFT, Phys. Rev. D 82 (2010) 025019 [arXiv: 1004.1179] [InSPIRE].

[89] P. Arnold, D. Vaman, C. Wu and W. Xiao, Second order hydrodynamic coefficients from 3-point stress tensor correlators via AdS/CFT, JHEP 10 (2011) 033 [arXiv:1105.4645] [INSPIRE].

[90] R. Emparan, C.V. Johnson and R.C. Myers, Surface terms as counterterms in the AdS/CFT correspondence, Phys. Rev. D 60 (1999) 104001 [hep-th/9903238] [INSPIRE].

[91] P. Kraus, F. Larsen and R. Siebelink, The gravitational action in asymptotically AdS and flat space-times, Nucl. Phys. B 563 (1999) 259 [hep-th/9906127] [INSPIRE].

[92] S. de Haro, S.N. Solodukhin and K. Skenderis, Holographic reconstruction of space-time and renormalization in the AdS/CFT correspondence, Commun. Math. Phys. 217 (2001) 595 [hep-th/0002230] [INSPIRE].

[93] R. Manvelyan, E. Radu and D.H. Tchrakian, New AdS non Abelian black holes with superconducting horizons, Phys. Lett. B 677 (2009) 79 [arXiv:0812.3531] [INSPIRE].

[94] G. Siopsis and J. Therrien, Analytic Calculation of Properties of Holographic Superconductors, JHEP 05 (2010) 013 [arXiv: 1003.4275] [INSPIRE].

[95] H.-B. Zeng, X. Gao, Y. Jiang and H.-S. Zong, Analytical Computation of Critical Exponents in Several Holographic Superconductors, JHEP 05 (2011) 002 [arXiv:1012.5564] [INSPIRE].

[96] M. Natsuume and M. Ohta, The Shear viscosity of holographic superfluids, Prog. Theor. Phys. 124 (2010) 931 [arXiv:1008.4142] [INSPIRE].

[97] J. Erdmenger, P. Kerner and H. Zeller, Non-universal shear viscosity from Einstein gravity, Phys. Lett. B 699 (2011) 301 [arXiv: 1011.5912] [InSPIRE].

[98] J. Erdmenger, P. Kerner and H. Zeller, Transport in Anisotropic Superfluids: A Holographic Description, JHEP 01 (2012) 059 [arXiv:1110.0007] [INSPIRE].

[99] J. Erdmenger, D. Fernandez and H. Zeller, New Transport Properties of Anisotropic Holographic Superfluids, JHEP 04 (2013) 049 [arXiv: 1212.4838] [INSPIRE].

[100] P. Basu, A. Mukherjee and H.-H. Shieh, Supercurrent: Vector Hair for an AdS Black Hole, Phys. Rev. D 79 (2009) 045010 [arXiv:0809.4494] [INSPIRE].

[101] M. Ammon, J. Erdmenger, V. Grass, P. Kerner and A. O'Bannon, On Holographic p-wave Superfluids with Back-reaction, Phys. Lett. B 686 (2010) 192 [arXiv:0912.3515] [INSPIRE].

[102] S.S. Gubser, F.D. Rocha and A. Yarom, Fermion correlators in non-abelian holographic superconductors, JHEP 11 (2010) 085 [arXiv: 1002.4416] [INSPIRE].

[103] E. Witten, Anti-de Sitter space, thermal phase transition and confinement in gauge theories, Adv. Theor. Math. Phys. 2 (1998) 505 [hep-th/9803131] [InSPIRE].

[104] M. Cvetič and S.S. Gubser, Phases of $R$ charged black holes, spinning branes and strongly coupled gauge theories, JHEP 04 (1999) 024 [hep-th/9902195] [INSPIRE].

[105] A. Chamblin, R. Emparan, C.V. Johnson and R.C. Myers, Charged AdS black holes and catastrophic holography, Phys. Rev. D 60 (1999) 064018 [hep-th/9902170] [InSPIRE].

[106] A. Chamblin, R. Emparan, C.V. Johnson and R.C. Myers, Holography, thermodynamics and fluctuations of charged AdS black holes, Phys. Rev. D 60 (1999) 104026 [hep-th/9904197] [INSPIRE]. 
[107] P. Mitra, Thermodynamics of charged anti-de Sitter black holes in canonical ensemble, Phys. Lett. B 459 (1999) 119 [gr-qc/9903078] [INSPIRE].

[108] S.S. Gubser and I. Mitra, The evolution of unstable black holes in anti-de Sitter space, JHEP 08 (2001) 018 [hep-th/0011127] [INSPIRE].

[109] S.S. Gubser and F.D. Rocha, The gravity dual to a quantum critical point with spontaneous symmetry breaking, Phys. Rev. Lett. 102 (2009) 061601 [arXiv:0807.1737] [INSPIRE].

[110] S.S. Gubser and A. Nellore, Low-temperature behavior of the Abelian Higgs model in anti-de Sitter space, JHEP 04 (2009) 008 [arXiv:0810.4554] [INSPIRE].

[111] S.S. Gubser and A. Nellore, Ground states of holographic superconductors, Phys. Rev. D 80 (2009) 105007 [arXiv:0908.1972] [INSPIRE].

[112] G.T. Horowitz and M.M. Roberts, Zero Temperature Limit of Holographic Superconductors, JHEP 11 (2009) 015 [arXiv:0908.3677] [INSPIRE].

[113] R.A. Konoplya and A. Zhidenko, Holographic conductivity of zero temperature superconductors, Phys. Lett. B 686 (2010) 199 [arXiv: 0909.2138] [INSPIRE].

[114] T. Nishioka, S. Ryu and T. Takayanagi, Holographic Superconductor/Insulator Transition at Zero Temperature, JHEP 03 (2010) 131 [arXiv:0911.0962] [INSPIRE].

[115] G.T. Horowitz and B. Way, Complete Phase Diagrams for a Holographic Superconductor/Insulator System, JHEP 11 (2010) 011 [arXiv:1007.3714] [INSPIRE].

[116] Y. Brihaye and B. Hartmann, Holographic superfluid/fluid/insulator phase transitions in 2+1 dimensions, Phys. Rev. D 83 (2011) 126008 [arXiv:1101.5708] [INSPIRE].

[117] P. Basu, J. He, A. Mukherjee and H.-H. Shieh, Hard-gapped Holographic Superconductors, Phys. Lett. B 689 (2010) 45 [arXiv:0911.4999] [inSPIRE].

[118] P. Basu, Low temperature properties of holographic condensates, JHEP 03 (2011) 142 [arXiv:1101.0215] [INSPIRE].

[119] A. Akhavan and M. Alishahiha, P-Wave Holographic Insulator/Superconductor Phase Transition, Phys. Rev. D 83 (2011) 086003 [arXiv:1011.6158] [INSPIRE].

[120] R.-G. Cai, H.-F. Li and H.-Q. Zhang, Analytical Studies on Holographic Insulator/Superconductor Phase Transitions, Phys. Rev. D 83 (2011) 126007 [arXiv:1103.5568] [INSPIRE].

[121] C. Wu, Angular Momentum Generation from Holographic Chern-Simons Models, arXiv: 1311.6368 [INSPIRE]. 